\section{Authors:}

6

\title{
Time to leave: Computations of when to end a social interaction depend on opportunity costs, depression, and loneliness
}

Anthony S. Gabay ${ }^{1,2,3}$, Andrea Pisauro ${ }^{1,2,3}$, Kathryn C. O'Nell ${ }^{3}$, Rosie Lynch ${ }^{3}$, Matthew A.J. Apps ${ }^{1,2,3,4}$

${ }^{1}$ Centre for Human Brain Health, School of Psychology, University of Birmingham

${ }^{2}$ Institute for Mental Health, School of Psychology, University of Birmingham

${ }^{3}$ Department of Experimental Psychology, University of Oxford

${ }^{4}$ Christ Church, University of Oxford

Dr. Matthew Apps, Centre for Human Brain Health, University of Birmingham, United Kingdom. E-mail: m.a.j.apps@bham.ac.uk

Dr. Anthony Gabay, Centre for Human Brain Health, University of Birmingham, United Kingdom. Email: anthony.s.gabay@gmail.com 


\section{Abstract}

9 Positive social relationships are vital for mental health. There is an ever-increasing understanding of 10 the cognitive and computational mechanisms that underlie how we process others' behaviours during 11 social interactions. Yet fundamentally many conversations, partnerships and relationships have to 12 end. However, little is known about how people decide when to leave. Theories of decision-making posit that people stop a behaviour in favour of another based on evidence accumulation processes, shaped by the value of alternative behaviours (opportunity costs). Do people compute evidence to leave social interactions based on the opportunity costs of connecting to others? Here, in a novel economic game, participants made decisions of when to leave partners in social environments with different opportunity costs for moving on. Across four studies we find that people leave partners more quickly when the opportunity costs are high, both in terms of the average generosity in the environment and the effort required to connect to the next partner. People's leaving times could be accounted for by a fairness-adapted evidence accumulation model, with a lower threshold for leaving in high opportunity cost social environments. Moreover, decisions to leave were modulated by depression and loneliness scores, which were linked to an interaction between the fairness of a partner and the opportunity cost of the social environment. These findings demonstrate the cognitive and computational processes underlying decisions to leave social interactions, and highlight that loneliness and depression may be linked to an atypical dynamic allocation of time to social interactions. 
Positive interpersonal relations are fundamental to mental health ${ }^{1-7}$. To understand what underlies positive social interactions, research in social psychology, neuroscience, psychiatry, and behavioural economics has examined how we react to others' behaviours during interactions. For example, economic games have been fruitful in examining the extent to which we punish others when they are being unfair, uncooperative or untrustworthy ${ }^{1-9}$. Yet much of this research ignores one of the most common responses to another's actions: we leave. In the real-world even if we feel unfairly treated, many reactions to others' behaviours such as punishment can be unwise, or simply not possible. For example, imagine being at a party where a work colleague starts making statements you disagree with. While you may feel tempted to rebuke them, this could have significant, negative personal consequences in the future. So instead, people often decide to end that social interaction. How do people evaluate that an interaction is declining in value, and decide when it is time to leave?

Despite almost all conversations, and many relationships, necessarily ending at some point ${ }^{17}$, little is known about how people decide when to leave a social interaction. Existing research has focused on what content leads to people leaving a conversation, the phrases used to end them, or examined how misaligned people's preferences are for the duration of an interaction ${ }^{17-20}$. However, few studies have examined the computational processes underlying how we ascribe value to a social interaction, evaluate that it is declining, and make a decision to move on from it. Strikingly, such decisions approximate problems studied in decision science and behavioural ecology about when animals stop one activity, in favour of another ${ }^{21,22}$. Therefore we propose that decisions to leave a social interaction should depend on similar decision-making mechanisms, and in particular on opportunity costs.

An opportunity cost is typically defined as the value of alternative, or foregone, activities one could be engaged $\mathrm{in}^{22-25}$. Theories of opportunity cost processes are particularly powerful because they make specific predictions about how to optimally allocate time $e^{21,24,26,27}$, rather than focusing on binary choices between options, as is more common in cognitive and decision sciences ${ }^{22,28,29}$. That is, the time you spend engaged in one activity is influenced by the value of the time you could spend in others. In doing so, they formalise the processes underlying when to leave an activity. Yet, such theories have rarely been applied to research in social cognition, and in particular to how people make decisions to leave a social interaction ${ }^{28}$. Here, we suggest that the quality of one's social environment shapes the opportunity cost of other possible interactions one could have, and thus how long people should spend voluntarily interacting with others.

Opportunity cost theories assume that people will allocate time to different behaviours in order to maximise a desired resource ${ }^{21,30-32}$. For example, imagine a bee foraging for pollen. As it remains at a 
flower, the amount of pollen left available declines. To maximise intake, it needs to decide when is the best time to leave and travel to the next flower. To do this optimally, it must compare the value of the pollen it is currently getting, to the average value of pollen intake it could get from other flowers in the environment - the opportunity cost. As such, in a rich environment with a high opportunity cost where lots of the other flowers give out lots of pollen, it should spend less time interacting with each flower than when the opportunity costs are low. Many species' behaviours conform to this principle of leaving sooner from locations in rich environments when collecting rewarding resources ${ }^{26,27,31-36}$. Human behaviours are also similarly sensitive to opportunity $\operatorname{costs}^{27,28}$, including for eye movements ${ }^{37}$, the allocation of cognitive control ${ }^{25}$, and time spent online ${ }^{38,39}$. Recent theoretical accounts suggest that opportunity cost based decision-making can be unified with computational and neural processes that underlie evidence accumulation ${ }^{40,41}$. That is, evidence is accumulated towards a decision to leave, which is triggered when a threshold is reached ${ }^{40,41}$. Therefore, we hypothesised that in richer social environments, people will end interactions with others more quickly due to changes in the evidence accumulation process.

Notably, psychiatric symptoms such as depression and loneliness are linked to less time being spent in positive social interactions, and poorer interpersonal relationships ${ }^{1,4,42}$. Risk factors for depression and loneliness include unhelpful cognitive processes at the individual-level, such as heightened sensitivity to others' threatening and unfair behaviour ${ }^{1,43-46}$. Yet there are also environmental risk factors, such as poorer neighbourhood cohesion and less supportive social networks, that affect who a person is likely to get the opportunity to interact with ${ }^{4,6,45,47,48}$. Strikingly, an opportunity cost account could provide a normative explanation for how much time people spend in social interactions and how they adapt this as the quality of the social environment changes. That is, in poor social environments it might be adaptive to tolerate slightly lower quality social interactions than in a richer one i.e. you might spend less time talking to a slightly disagreeable colleague at a party where everyone else is much more agreeable. Thus, maladaptive opportunity cost processes underlying decisions to leave may be a hallmark of increased loneliness and depression.

We developed a novel paradigm to test the notion that people decide to end social interactions that are declining in value depending on opportunity costs, and evidence accumulation processes. Unlike other economic games, in this task participants' only decision was when to leave one social interaction in favour of another (Fig.1; Supplementary figure 1). Specifically, participants were connected in oneto-one interactions with a partner. Each interaction took the form of repeated decisions from the partner about how much of a pot of money to share with them, similar to repeated rounds of the dictator game ${ }^{49}$. When a participant no longer wished to be connected to that partner they could voluntarily leave, waiting eight seconds to connect to a new, different partner. To change the value of 
interacting with different partners, we manipulated their fairness - the proportion of the pot they were sharing ${ }^{10,11,13,50,51}$. Some partners' fairness decayed at a faster rate than others over time - similar to decays in fairness observed in multi-round economic games and in real-world social interactions ${ }^{52,53}$. Participants engaged in these interactions during five-minute periods in different 'social environments', which differed in the opportunity costs of moving on. In studies one, two and four we manipulated the 'average generosity', changing the proportion of fair or less fair partners in each environment. In study three we altered how much effort needed to be exerted during the eightsecond delay in order to connect to the next partner $28,54,55$. This created low opportunity cost environments, where the majority of partners were unfair or where it was effortful to connect the next partner, and high opportunity cost environments where most partners were fair or it was low in effort to connect to a new partner.

Across four studies we demonstrate and replicate that the length of time people spend interacting with others increases when a partner is fairer, but also increases in low quality social environments with lower opportunity costs. Moreover, we found that distributions of time spent connected to partners could be accounted for by a fairness-adapted evidence accumulation model, with a higher threshold when the opportunity costs were low. Lastly, self-reported depression and loneliness interacted with the fairness of the partner, and the generosity of the environment, influencing leaving times. These findings support the notion that during social interactions we build evidence towards decisions to leave in a manner that is shaped by the opportunity cost of the alternatives. Moreover, aberrant processing of the variables that underlie decisions to leave may be a marker of the interpersonal challenges related to variability in loneliness and depression leading to less time being allocated to positive social interactions. 
120 To test an opportunity cost based account of how people dynamically allocate their time to interacting

121 with different people and make decisions to leave social interactions, we conducted four studies. In

122 each study, participants were connected to an anonymous partner and made decisions of when to

123 leave that partner to connect to another (Fig.1, Supplementary Fig.1). While connected, they saw how

124 the partner decided to share pots of money of different total magnitudes, with the fairness of these

125 decisions - the proportion of the pot shared - decaying at different rates (creating fair and unfair

126 partners). Importantly, pot sizes were manipulated, such that on average, all partners were equal in

127 the total monetary value that could be obtained while being connected to them. As such, any decision

128 to leave was economically sub-optimal, reduced the amount of money that would be earned, and thus

129 indicated sensitivity to fairness. During the task, participants spent multiple blocks lasting five minutes

130 interacting with partners in different social environments. Across blocks, we manipulated these

131 environments to create high or low opportunity costs for switching between partners. This was either

132 by altering the average generosity - the proportion of fair and unfair partners (Studies 1, 2 \& 4) - or

133 the effort required to switch partners (Study 3). Across studies we examined participants' "leaving

134 time", the amount of time they spent connected to partners, as a function of the fairness of the

135 partner and quality of the environment. (Fig. 1 and Methods). An opportunity cost based account

136 predicts that people should spend more time with fairer partners, but should leave partners sooner

137 when in high quality (generous or low effort) environments (Fig.1C). 


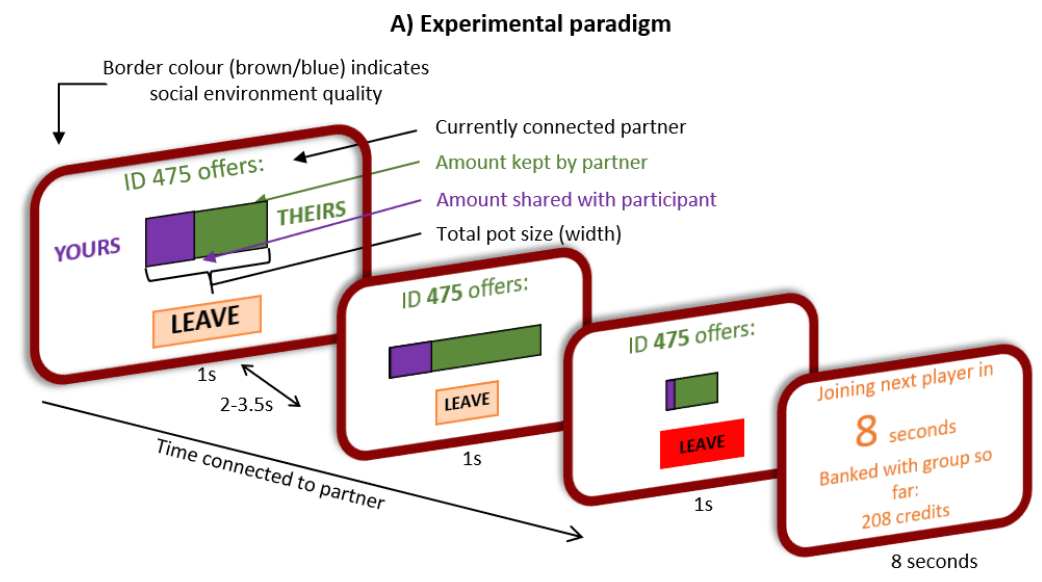

B) Fairness of partners decays over time (Study 4)

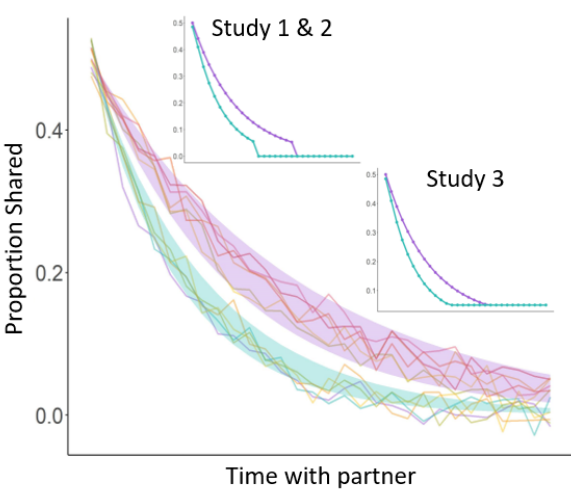

C) Hypothesised effects of opportunity costs on social interaction durations

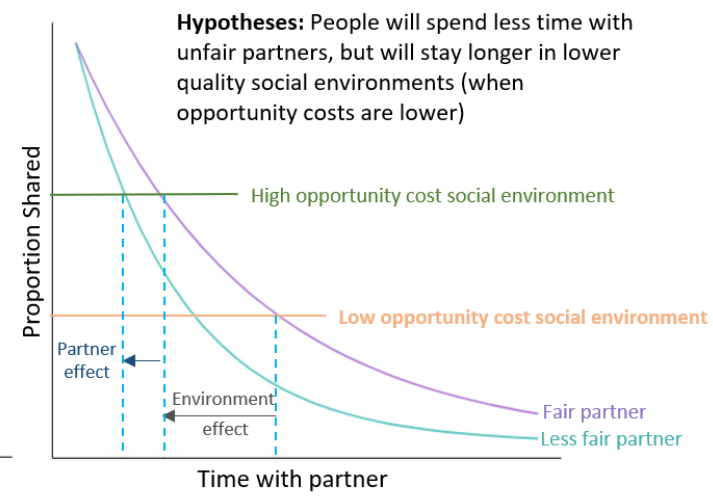

Figure 1: A) Experimental paradigm. Participants were connected with partners (indicated by numerical ID numbers). Partners made decisions about how much to share out of different pots of total credits of different sizes, indicated by the width of a bar on the screen. The amount being shared was purple, and amount kept by the partner shown in green. Participants' task was to decide when to leave a partner to connect to another. When participants chose to leave, they experienced an eight second delay during which they were shown the amount of credits collected in the environment so far. Participants joined different virtual "groups" of potential partners for five-minute blocks, creating different social environments. This information was indicated by a coloured border for the entirety of blocks and an instruction screen between blocks. For variations in design for studies $1-3$, see supplementary materials. (B) The decisions by partners were experimentally controlled with different rates of decline in fairness - the proportion shared. The lines represent examples of proportions shared by partners over time. Studies 1,2 and 3 (inset) had only two types of partner. Study 4 had multiple partners, with noise surrounding the rates of decay of fairness. The purple shaded area represents proportions shared from "fair" partners, the green shaded area represents proportions shared by unfair partners. (C) An opportunity cost account predicts that people will leave sooner from less fair (teal line) than fair (purple) partners, but also that it will depend on the opportunity cost afforded by the social environment. To manipulate opportunity costs participants for studies 1, 2 and 4, environments differed by their average generosity, determined by the proportion of fair or unfair players included in the virtual group. In study 3 , they differed by the amount of effort (a high or low number of button presses) to be completed before being connected to a new partner. When effort was low, or average generosity high (green line), the opportunity cost was higher and thus it is predicted people would leave partners sooner than when the opportunity cost was low (orange). 
In Study $1(n=19)$ and Study $2(n=25)$, the social environment was manipulated by controlling the proportion with which participants would encounter two different types of partner, who differed by the rate at which the fairness of their sharing decisions decayed. There were two types of environment: high and low generosity, which differed by the ratio of fair to unfair partners $75: 25$ high; 25:75 low). To test our hypotheses that participants would spend less time interacting with unfair partners than fair partners, and that they would spend less time with partners in a high generosity environment than a low generosity environment, we carried out a linear mixed-effects analysis. This model comprised time spent interacting as the outcome variable, and partner-type (fair or unfair), environment generosity (high or low), and their interaction, as predictors. We predicted main effects of partner-type and environment, but no interaction, in line with opportunity cost theories ${ }^{27,54}$.

In Study 1 (Fig.2A), we found significant main effects of partner-type and environment, with participants spending less time interacting with unfair partners than fair partners, and less time interacting with partners in a high generosity environment than in a low generosity environment (partner-type: $b=-13.86, S E=1.77, t_{(17.88)}=-7.82, p<0.001 ; X^{2}{ }_{(1)}=61.77, p<0.001$; environment: $b=$ $\left.3.50, S E=1.53, t_{(17.95)}=2.28, p=0.035 ; X^{2}{ }_{(1)}=5.37, p=0.020\right)$. There was also a significant interaction (partner-type x environment: $b=-3.53, S E=1.35, t_{(909.74)}=-2.26, p=0.009 ; X^{2}{ }_{(1)}=6.88, p=0.009$ ), such that the difference between high and low generosity environments was statistically significant for fair $\left(b=-5.27, S E=1.67, t_{(25)}=-3.157, p=0.004\right)$, but not unfair $\left(b=-1.74, S E=1.69, t_{(25.9)}=-1.031, p=\right.$ $0.3121)$ partners.

Partner-type and environment effects remained significant even when excluding decisions that were made after fairness had decayed to zero (Supplementary results and Supplementary Table 2). In addition, we also performed a similar mixed-effects model with the same predictors, but using the fairness (proportion shared) at the time of leaving as the outcome variable. We found the same pattern of results, with main effects of partner-type and environment, but no interaction between them (Supplementary results and Supplementary Table 3). Notably this behaviour was not economically rational, as leaving a partner led to an eight second period where no reward was being collected. The patterns of behaviour outlined above therefore also led to people earning less money from unfair partners (by leaving them earlier) and earning less money in the high opportunity cost environments (Supplementary results and Supplementary Table 4).

In Study 2 (Fig. 2B) we sought to replicate these findings, using a sample size $(n=25)$ selected based on power calculations from Study 1 (Methods). We replicated the main effects of partner and social environment (partner-type: $b=-13.38, S E=1.54, t_{(23.74)}=-8.69, p<0.001 ; X^{2}{ }_{(1)}=75.48, p<0.001$; 
environment: $\left.b=3.75, S E=1.42, t_{(23.20)}=2.64, p=0.015 ; X^{2}{ }_{(1)}=6.97, p=0.008\right)$, but found no interaction (partner-type $x$ environment: $b=-0.64, S E=1.41, t_{(1166.58)}=-0.46, p=0.649 ; X_{(1)}=0.21, p$

$174=0.649)$. Partner-type and environment effects also remained significant even if decisions after 175 fairness had decayed to zero were excluded (Supplementary results and Supplementary Table 2). A 176 mixed-effects model with fairness at the time of leaving as the outcome variable showed the same 177 pattern of results, with main effects of partner-type and environment (Supplementary results and 178 Supplementary Table 3). These patterns of behaviour therefore also led to people earning less money 179 from unfair partners - by leaving them earlier - and earning less money in the high-opportunity cost environments (Supplementary results and Supplementary Table 4).

181 Taken together, these studies suggest that people's decisions to leave a social interaction are made 182 based on both how fair that person is behaving, as well as the opportunity costs of the social environment, with more generous environments favouring leaving a partner sooner. 
Participants spend less time interacting in high generosity and low effort groups than low generosity or high effort groups

A Study 1

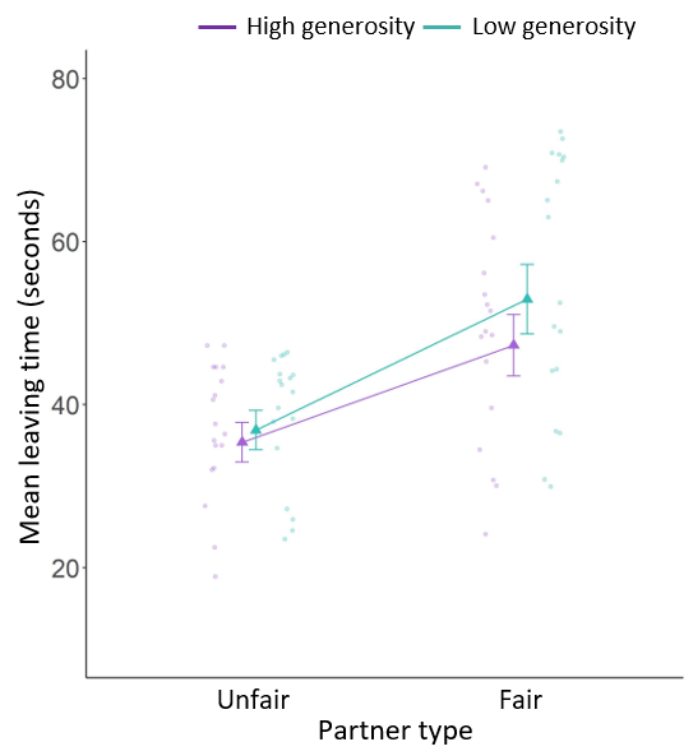

C Study 3

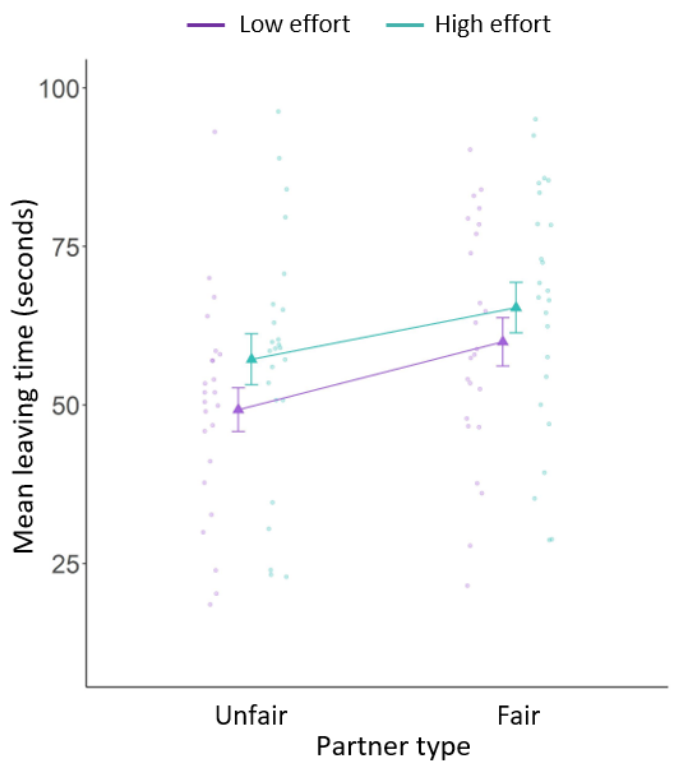

B Study 2

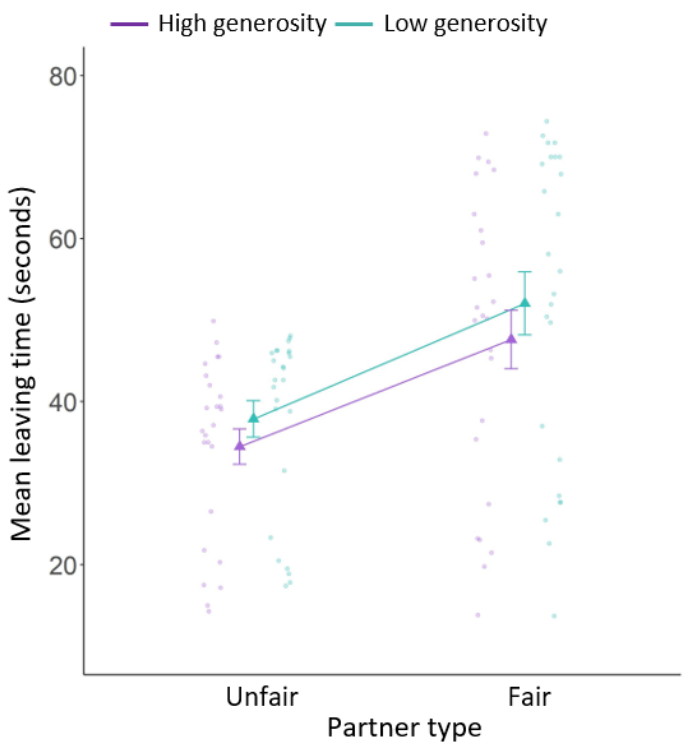

D Study 4

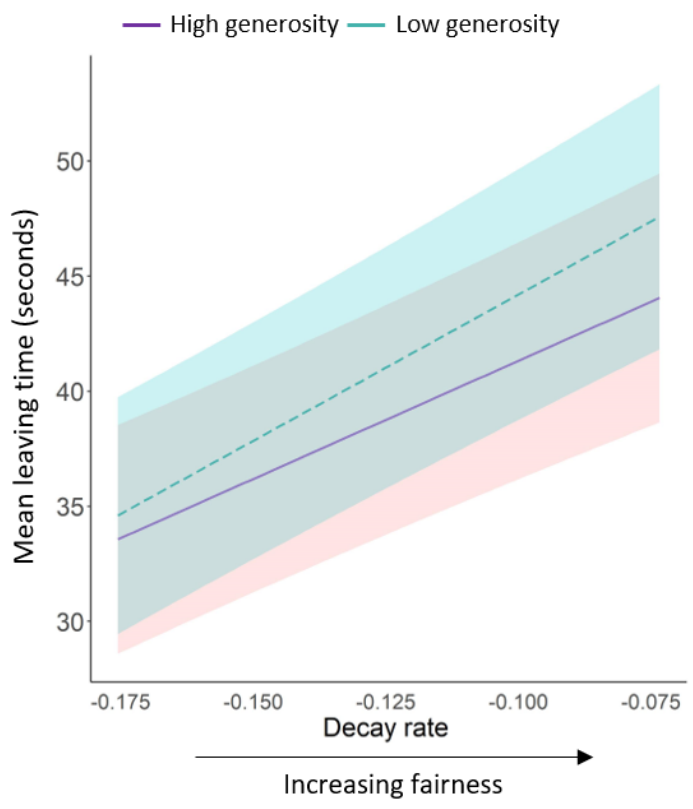

Figure 2: Mean leaving times - time spent connected before deciding to leave - with partners of different types and in the different quality social environments. There were significant main effects of partner-type and environment in all four studies. In all studies participants spent more time connected with fairer partners, but less time with partners when the opportunity cost of the environment was high (i.e. when the group was generous or when it was low effort to connect to another partner). Means from study 1 (A), study 2(B) where the social environment quality was defined by average generosity, high (purple) and low (teal). In study 3 (C) the social environment quality was defined by the effort - the number of button presses required to connect to the next partner. For study 4 (D), partner type was a continuous variable, with shaded regions representing $95 \% \mathrm{Cls}$. Points represent individual participant means, triangles represent the summary means with error bars of $\pm 1 S E$. 
Previous research has suggested that opportunity costs are influenced not only by the average benefits available from alternatives in the environment, but also, the costs that must be incurred to switch from one activity to another ${ }^{55}$. When the cost to leave and explore is higher, the value of alternatives is reduced. This is particularly true for the effort of travelling to seek out alternatives ${ }^{54-58}$. Thus, in Study 3 we examined whether the effort required to switch from one partner to another influenced how long people spent connected. We tested the hypothesis that participants would spend less time interacting with partners when there were less effort costs to switch between interactions. As with Study 1 and Study 2, there were two different types of partner (fair and unfair), but in this study the ratio was 1:1 in both environments. As such, there were no differences in average generosity. Instead, effort costs were introduced to the task, which differed between environments. When participants left a partner, they were required to exert effort during the eight second delay. This effort was operationalised as high or low numbers of repeated button presses, calibrated to a participant's own maximum numbers of button presses. Each environment required either the high or low effort, defined as $80 \%$ and $20 \%$ of the participants' maximum, respectively.

201

Once again we found significant effects of partner-type and environment (Fig.2C). Participants spent less time interacting with unfair partners than fair partners (partner-type: $b=-9.10, S E=1.38, t_{(812.58)}$ $\left.=-6.60, p<0.001 ; X_{(1)}^{2}=44.30, p<0.001\right)$, but also spent less time connected to partners when less effort was required to switch between them (environment: $b=6.13, S E=1.38, t_{(812.68)}=4.45, p<0.001$; $\left.X^{2}{ }_{(1)}=19.53, p<0.001\right)$. There was no significant interaction $\left(b=1.45, S E=2.75, t_{(812.32)}=0.53, p=\right.$ $\left.0.599 ; X^{2}{ }_{(1)}=0.28, p=0.599\right)$. A mixed-effects model with fairness at the time of leaving as the outcome variable showed the same pattern of results, with main effects of partner-type and environment (Supplementary results and Supplementary Table 3). Thus, participants spent less time with less fair partners, but spent longer interacting with a partner if it was effortful to connect to another. In Study 4, as well as replicating the effects from the previous studies there were three additional aims: (i) To better represent real-life social behaviours, we included multiple different partner types, rather than just two (fair and unfair). We created a continuum of 17 decay rates describing the rate at which partners' sharing decisions became less fair. Additionally, we added noise around the decay curve so the change in fairness was less predictable and to make each partner's pattern of decisions unique (Fig.1 B). (ii) to test hypotheses relating to depression and loneliness we collected a larger 
sample $(n=81)$ in an online version of the task, and in addition to completing the task, we had participants complete the Depression, Anxiety and Stress Scale (DASS) ${ }^{3}$ as well as the De Jong Gievald loneliness scale $(D G L S)^{59}$, (iii) we also included additional blocks, in order to increase the number of samples per participant. This allowed us to fit evidence accumulation models to data without overfitting to individual data or pooling data across participants (see below).

We initially defined a mixed-effects statistical model equivalent to those in Study 1 and Study 2, except with the partner-type (fair/unfair) replaced with the continuous "decay rate" of the partner. Replicating the previous results, there was a main effect of both the decay rate of the partner and environment (partner: $b=3.80, S E=0.43, t_{(65.13)}=8.79, p<0.001 ; X_{(1)}^{2}=76.23, p<0.001$; environment: $\left.b=2.22, S E=0.82, t_{(58.46)}=2.72, p=0.009 ; X^{2}{ }_{(1)}=7.33, p=0.007\right)$, with no significant interaction $(b=$ $\left.0.81, S E=0.44, t_{(5362.64)}=1.83, p=0.068 ; X^{2}{ }_{(1)}=3.33, p=0.068\right)$. A mixed-effects model with fairness at the time of leaving as the outcome variable showed the same pattern of results, with significant effects of partner and environment (Supplementary results and supplementary Table 3).

Next, we included scores from the DASS-21 into the mixed-effects model with leaving time as the outcome variable. The DASS-21 has three subscales (depression, anxiety and stress). Six participants did not go on to complete the questionnaires, making the sample size for this analysis $n=75$. Model comparison showed that a mixed-model with just the depression scores had a better fit than those additionally including anxiety and stress. As such the model presented here included the main effects of partner type (decay rate), environment, and depression, as well as the two-way and three-way interactions. This model showed the main effects of partner-type and environment as that presented above (partner: $b=3.85, S E=0.47, t_{(60.02)}=8.27, p<0.001 ; X^{2}{ }_{(1)}=68.56, p<0.001$; environment: $b=$ $\left.2.03, S E=0.85, t_{(52.29)}=2.38, p=0.021 ; X^{2}{ }_{(1)}=5.68, p=0.017\right)$. Indluing depression in the model resulted in a change in the interaction effect, with the two-way partner-by-environment interaction becoming signifcant $\left(b=0.86, S E=0.46, t_{(4896.88)}=1.89, p<0.059 ; X^{2}{ }_{(1)}=4.00, p=0.046\right)$. Additionally, we found a three-way interaction between partner, environment, and depression score (Fig.3; $b=1.04, S E=$ $\left.0.48, t_{(4885.67)}=2.18, p=0.030 ; X_{(1)}^{2}=4.73, p=0.030\right)$. The main effect of depression, and all other twoway interactions were non-significant ( $p s>0.05$ ). We found exactly the same pattern of results when including loneliness scores instead of depressions scores. There was a three-way interaction between partner, social environment, and loneliness score $\left(b=0.56, S E=0.26, t_{(4892.66)}=2.18, p=0.030 ; X^{2}{ }_{(1)}=\right.$ $4.73, p=0.030$ ). The main effect of loneliness, and all two-way interactions were non-significant ( $p s>$ 0.05). We were unable to include both depression and loneliness in the same statistical model due to their high correlation (Spearman's rho $=0.59$ ). 
Post-hoc analyses indicated that there were partner-by-depression and partner-by-loneliness interactions in the low generosity environment, but not in the high generosity environment (Fig.3). In the low generosity environment, participants with higher depression (and loneliness scores) spent less time interacting with unfair partners than those with low depression (or loneliness) scores. Moreover, participants with higher depression and loneliness scores spent a similar amount of time interacting with unfair partners in both types of environment, which was not the case for participants with low depression scores. Notably this effect could not be explained by behaviour being more economically rational, as overall earnings for the task were not predicted by depression or loneliness scores (Supplementary results and supplementary Table 5).

As such, in line with our hypotheses, these results suggest that depression and loneliness are linked to atypical decisions to leave a social interaction, and in particular to atypical integration of the fairness of someone's behaviour with information about the opportunity costs of the social environment.

A

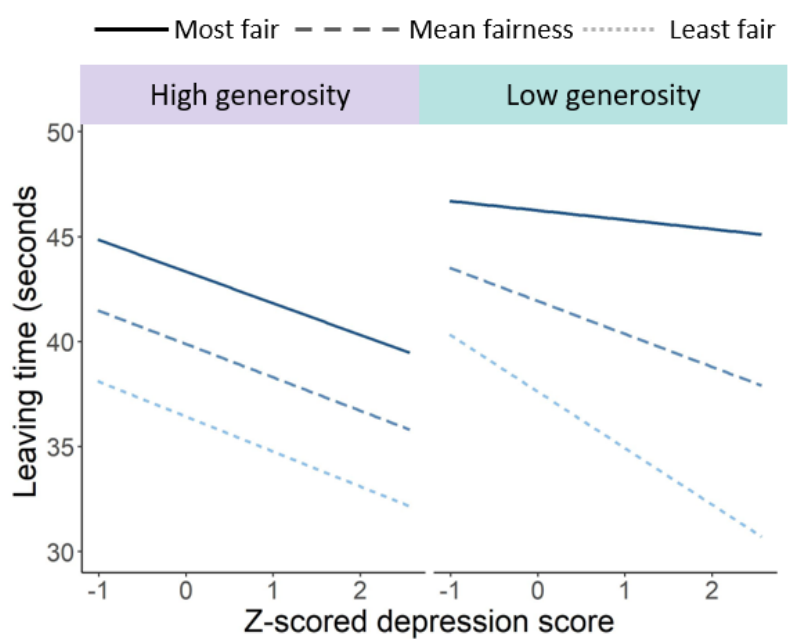

B

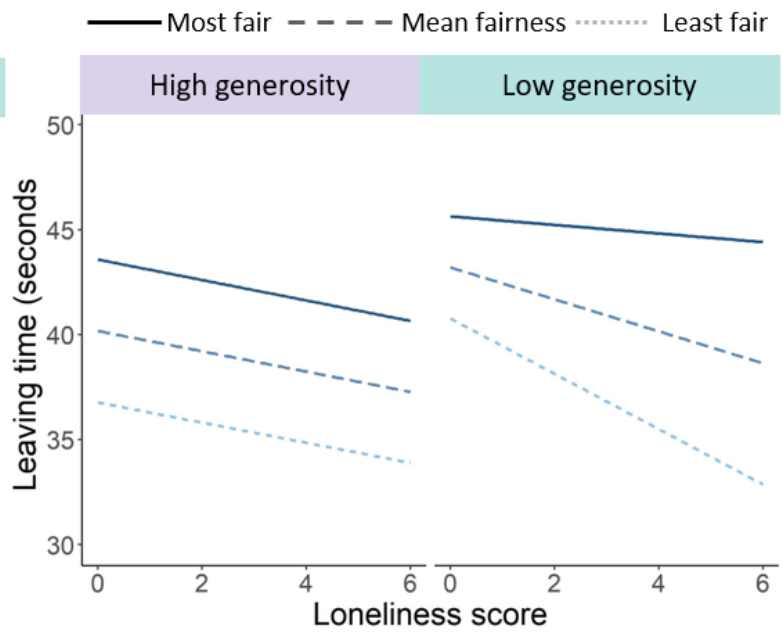

263

264

265

266

267

268

269

Figure 3: Study 4 results. A) Depicts the significant three-way interaction between partner-type, environment, and depression B) Depicts the three-way interaction between partner-type, environment, and loneliness. In both cases, in low generosity environments, participants with higher self-reported scores spent less time interacting with more unfair participants (three-way interaction $p=0.030$ for both measures). Thus, higher depression and loneliness ratings were linked to different sensitivity to the fairness of partners and the quality of the social environment on decisions to leave social interactions. 'Most fair' and 'least fair' refer to mean fairness plus 1SD and mean fairness minus $1 S D$, respectively.

A fairness-adapted evidence accumulation model accounts for decisions to leave 
To better understand the computational processes underlying how people evaluate the fairness of a social interaction with information about the opportunity costs afforded by the social environment, we modelled decisions to leave partners as an evidence accumulation process using an adapted drift diffusion model (DDM). This model assumed that participants are constantly and noisily accumulating evidence over the course of the interaction, and that the decision to leave is triggered when the amount of evidence reaches a threshold level (Fig. 4) $)^{40}$. The evidence accumulation process is described by four parameters (see Methods for full details): i) an initial starting value (bias); ii) the threshold to be reached; iii) the drift rate, which describes the rate of evidence accumulation; iv) the noise parameter, which describes how noisy the evidence accumulation is. We compared the fits of a standard model (Standard-DDM), which was naïve to the experimental manipulations, to two other models. In the first, the drift in evidence accumulation was modulated by the fairness of each of the partner's sharing decisions (Fairness-DDM). In the second, the magnitude of the reward being shared by the partner at each decision, rather than fairness, was used to modulate the drift in evidence accumulation (Reward-DDM).

We fit each of these three models to each participant's distribution of leaving times in Study 4, separately for each social environment. Model comparison (combined BIC across the two environments) revealed that the Fairness-DDM was a better fit to leaving time distributions than the Reward-DDM and Standard-DDM (Fig.4b). To further assess the quality of fit of the winning model, we computed correlation coefficients between the average leaving time from the data and the winning model for all participants and for each environment. The Spearman's rho was 0.98 and 0.99 for the high and low generosity environments, respectively, indicating good fit. In simulated data (Fig.4c), the Reward-DDM failed to account for the fairness of partners, and the Standard-DDM did not predict an environment effect (Supplementary results). In contrast, the Fairness-DDM was able to capture both partner-type and environment effects present in the statistical analysis of the data above. When running a linear mixed-effects model on the simulated data from the winning FairnessDDM, we found main effects of partner-type and social environment, (partner-type: $b=-6.54, S E=$ $0.13, t_{(161900)}=-49.827, p<0.001 ; X^{2}{ }_{(1)}=3877.81, p<0.001 ;$ environment: $b=1.66, S E=0.13, t_{(161900)}=$ $12.734, p<0.001 ; X_{(1)}^{2}=688.65, p=0.001$; see Supplementary results and Supplementary Table 4 for the other models).

If people were making decisions to leave in line with an evidence accumulation model, but adapting behaviour to the quality of the social environment, we would expect features - the parameter weights - of the model to differ between environments. In line with this, we found a higher threshold parameter in the low generosity social environment compared to the high generosity social environment $\left(X^{2}{ }_{(1)}=3.93, p=0.048\right)$. None of the other parameter values differed across social 

environments (Supplementary Figure 3). This suggests that people required more evidence to be 308 accumulated before making a decision to leave in a poor social environment when the opportunity 309 costs were low. 


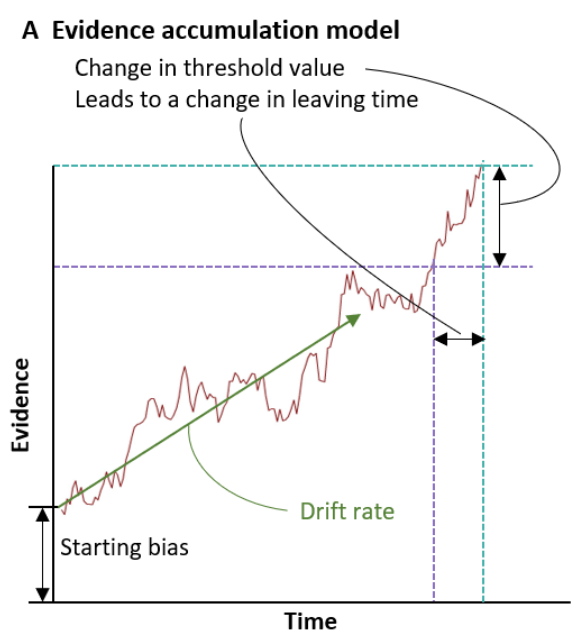

B Model comparison

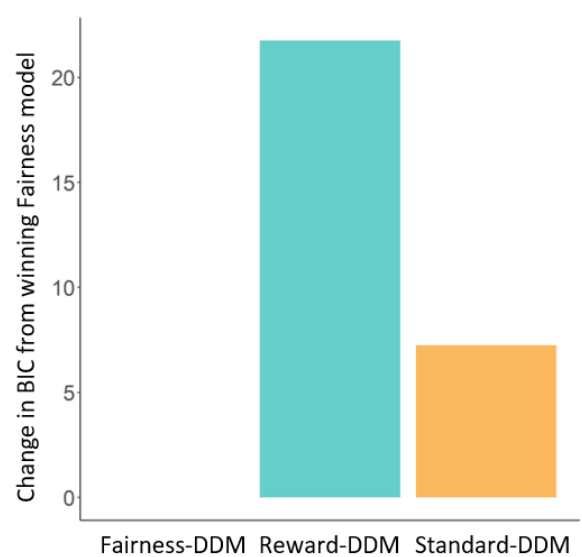

C Comparing real data to simulated data
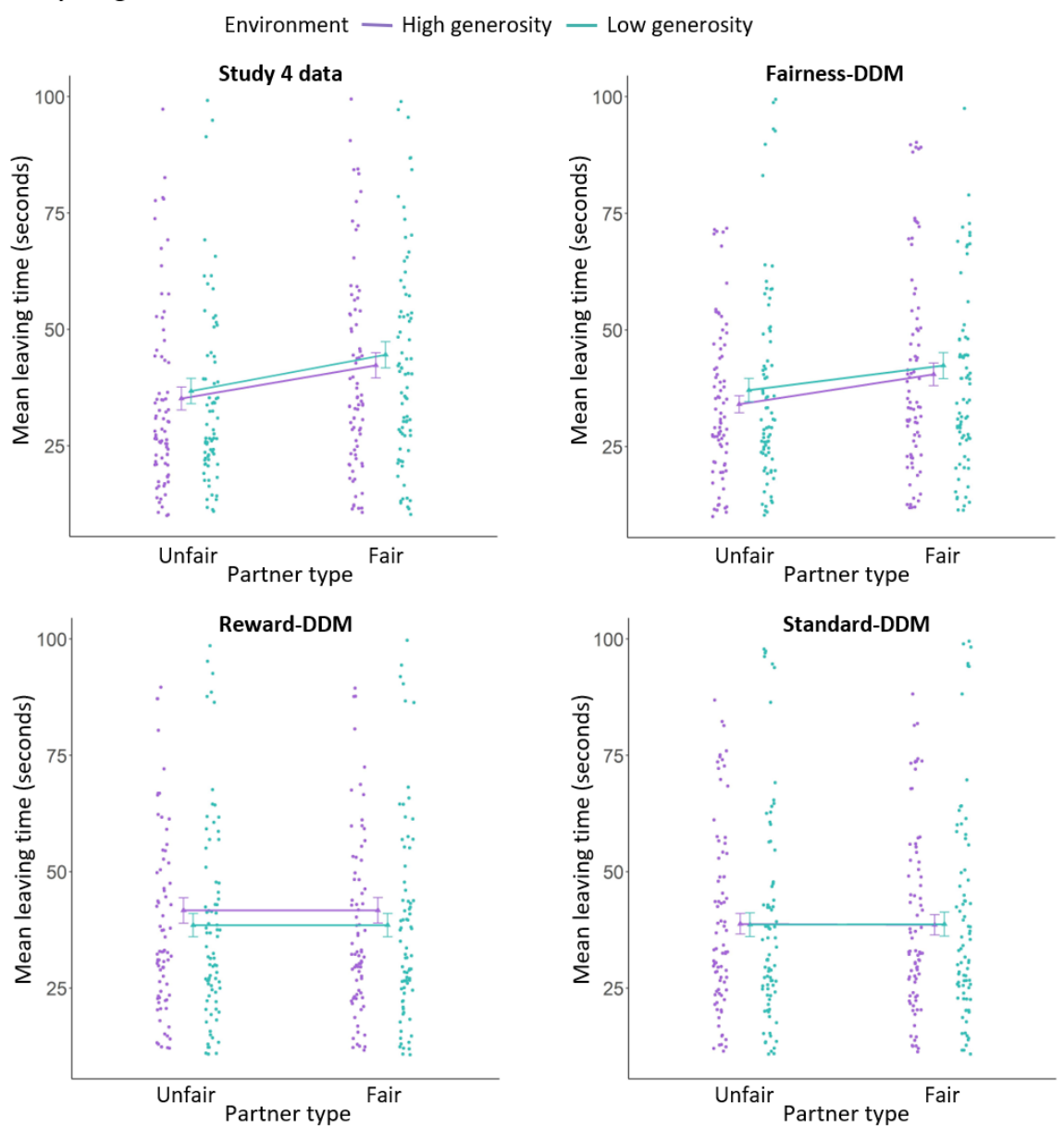

Figure 4: A) Schematic Representation of evidence accumulation models. In all models evidence accumulated to leave the social interaction over time. The fairness and reward DDMs adapted this accumulation, with the drift multiplied by reward obtained (reward) or proportion or shared (fairness) at the time of each decision by the partner B) Model comparison of the three competing models $(\mathrm{BIC})$ showed that the fairness model was best able to explain behaviour compared to the reward and standard DDMs C) Plots of the simulated data for each of the three models compared against data from study 4 (top left). Dots represent estimated/actual mean for a participant. Only the Fairness-DDM replicated the effects of partner-type and environment on simulated leaving times. Triangles represent estimated/actual mean across participants. Error bars represent SEM. 
We often have to decide whether to leave social interactions. Here we tested the hypothesis that deciding how long to spend in social interactions depends on how fairly we are treated, as well as the opportunity costs of moving on to other people in the environment. To do so, we manipulated the decay of how fairly participants were treated, and the quality of the social environment, in a novel economic game. Across four studies we show that people spend more time with fairer partners, but also spend more time with partners in poor social environments when opportunity costs are low, both when the environment quality was determined by the average generosity of other people and by the effort to connect to another person. In addition, leaving times were related to depression and loneliness, with scores on these measures linked to an interaction between how fairly a partner treated the participant and the quality of the environment. We were also able to capture the computations underlying decisions to leave using an adapted drift diffusion model: the evidence to leave reflected the decaying fairness of the interacting partner, with changes in the quality of the social environment reflected by a different threshold needed to be reached before leaving. Thus, ending a social interaction may rely on evidence accumulation processes that reflect the opportunity costs of moving on in different social environments Importantly, our results suggest that the length of time people spend in an interaction differs as a function of the quality of the social environment, and thus the opportunity cost. This was regardless of whether the opportunity cost was manipulated through changing the average generosity of the environment, or through manipulating how hard people would have to work to connect to another person. These findings align with recent research showing the importance of opportunity costs when making other types of decisions, such as whether to exploit a current location or travel and explore elsewhere to obtain rewards $s^{26,27,36}$ or how to allocate time to visual searches of social stimuli ${ }^{37,60}$. However, unlike in other experiments, in this task participants were sacrificing rewards when making decisions to leave social interactions. This suggests that opportunity costs apply to the value ascribed to the resource one is trying to maximise and not to reward value per $\mathrm{se}^{61,62}$. In our experiment the resource being maximised was the time spent in positive, fair, social interactions. Thus, our results suggest that opportunity cost principles can be applied to a much broader range of social decisions, including those that impact how much time we spend in social interactions ${ }^{28,61}$.

Our results also suggest that at the mechanistic level, similar neural and computational processes for other types of decision problems may also underlie how people end social interactions. We showed that choices in the task could be fitted with an adapted DDM, in which evidence to leave drifted towards a threshold, which when reached, triggered a decision to leave. The parameters used in this 
model, quantifying a starting bias in evidence, a threshold boundary, the rate of drift of evidence, and noise, are standard within evidence accumulation models ${ }^{63-65}$. Such models have been used to understand the psychological and brain processes guiding perceptual, economic and social decisions, but not previously to how people decide to leave social interactions ${ }^{64,66}$. Although this suggests that there may be normative, domain-general mechanisms that underlie such decisions, there were key differences in the model to a standard DDM. In particular the model was accumulating across the entire time connected to another player, but constantly changing as the fairness of the decisions of the partner changed. This model did better than a DDM in which evidence was simply accumulating over time, indicating that fairness may be a type of evidence that can be accumulated over longer timescales, as has been shown for rewards ${ }^{40,41}$. Moreover, by fitting this model separately to the different social environments, we could show a higher threshold when the model was fitted to the poorer social environment than the richer i.e. when opportunity costs were low. Taken together, these results suggest that decisions to leave a social interaction are contingent on the build-up of evidence, which depends on how much value is currently being ascribed to the social interaction (i.e. how fair it is). However, decisions change depending on the quality of the social environment. Higher quality environments create a lower decision threshold, and thus less accumulated evidence of unfair behaviour is needed before leaving.

A plethora of existing work has shown that people value fairness, and will lose out on economic rewards, in favour of being treated fairly ${ }^{10,51}$. In this respect, our participants' behaviour was consistent with that found in economic games. However, existing work had typically examined fairness as a static property, showing that people would punish others at a certain level of disadvantageous inequity - when an amount offered to ourselves in an economic game falls somewhere below $50 \%{ }^{10,11,15}$. Although some previous studies suggest that the context of a social interaction can influence how likely people are to punish unfair behaviour ${ }^{67-69}$, we are unaware of any previous study that directly manipulated the opportunity cost, nor examined how people decide when to leave a social interaction. In doing so, we show that fairness is not processed in an absolute manner. Instead, our results suggest that people adapt how fairly they are willing to be treated based on the average behaviour in a social environment. Indeed, we showed that people would stay longer, and that they would tolerate a lower level of fairness, in an environment where most people were less generous. Such findings suggest that people are moral relativists when it comes to fairness ${ }^{70,71}$. That is, how unfair they perceive an act to be changes based on how likely it is that other people would be similarly unfair.

Changing one's principles, such as what one considers as fair behaviour, based on what is the social norm can have potentially wide social and moral consequences ${ }^{71}$. However, our results suggest this 
behaviour may be typical and adaptive when it comes to allocating time to interacting with different people. Strikingly, we found that decisions to leave social interactions correlated with depression and loneliness. Higher depression and loneliness scores related to atypical patterns of behaviour, particularly in poorer social environments. Higher scores on these measures related to staying longer with fairer partners but less time with the least fair people in the poor environment relative to the rich. As in this task the fairness of partners decayed over time, this suggests that high depression and loneliness may be linked to a tendency to favour staying with individuals who were originally fair, but are no longer acting fairly, specifically in poor environments. Such findings may provide a mechanism for understanding why depression and loneliness have been linked to greater sensitivity to unfair behaviour ${ }^{43,44}$, but also risk factors that impact on the quality of social environment ${ }^{1,6}$. In particular our results highlight that a heightened sensitivity to unfair behaviour and being in a poor social environment may be interacting risk factors, leading to the perception of poor social relationships, which is common in depression and chronic loneliness ${ }^{43,45,47}$. As such, our results suggest that being appropriately sensitive to unfair behaviour, but also being a moral relativist in one's behaviour and adjusting to the quality of the social environment is adaptive, and may contribute to mental health. Moreover, these results highlight that interpersonal deficits linked to depression and loneliness may be better understood using decision theories that account for the dynamic allocation of time to social activities.

Recent work has sought to examine when people choose to end natural conversations, both in the lab and in real life, and reported that the preferred time to end a conversation rarely aligns across interacting partners ${ }^{72}$. Although many features of the content of conversations will influence their value that go beyond the fairness manipulation deployed in this experiment, our results point to a potential computational explanation for a lack of alignment. Specifically, as noted above, our results point to DDM-related processes underlying such decisions, which also include noise in the evidence accumulation. Moreover, they point to different thresholds being set when one's opportunity costs differ. As such, the chances of two people valuing the conversation equally, having similarly valued alternative opportunities, and aligned random noise in the accumulation of evidence towards ending the conversation are vanishingly small. Therefore, we suggest that the combination of all of these factors make ending a conversation at a mutually agreed point fraught with challenges ${ }^{20}$, even before one considers wider social rules, hierarchies, and the challenges with trying to infer the intentions of others.

Social interactions do not happen in isolation, and throughout our life we are faced with choices of joining and leaving social relationships, be they friendships, romantic partnerships, work collaborations, or simply ending a conversation. Our results show that the quality of a social 

environment - its opportunity costs - are crucial for determining when people make the decisions to leave. Such processes appear normative, and guided by evidence accumulation processes, with atypical consideration of opportunity costs a potential mechanism underlying disrupted social relationships in depression and the chronically lonely.

419

420

421

422

423

424

425 
428175 participants (mean age $=27.6(S D 7.6)$, range $18-51 ; 62 \%$ F) were recruited over four studies (see

429 Supplementary Table 1 for demographics broken down by study). All studies were approved by The 430 University of Oxford Central Research Committee (Studies 1 - 3 reference number R6061/RE001; Study 4 reference number R59122/RE001). Informed consent was obtained from all participants. In studies $1-3$, participants were recruited through an Oxford University participant database. In Study 4, participants were recruited through the online platform Prolific (www.prolific.co). Participants were compensated a flat rate for their time and were also told that they would receive a bonus based on their responses in the task. However, all participants were paid the bonus, to ensure remuneration was ethical, and not based on participants being deceived by the fairness manipulations in the study. The amounts paid to participants were equal across participants in each study (f14).

Experimental paradigm

\section{General task structure}

In our task we had participants interact with virtual partners in different environments with unlimited potential partners. Participants' task was to decide when to leave a partner, and wait to be connected to another. We manipulated the 'quality' of both partners and of the environments, to examine whether people make decisions based solely on the quality of an ongoing social interaction or whether they also consider the opportunity cost of other potential partners. Given the consistent finding of its importance to healthy social interactions, we used fairness as a proxy for the value of interacting with a partner ${ }^{10,11,13,50,51}$. The general structure of the task was consistent across all four studies.

Participants were instructed that they were virtually joining different groups of people for a total of five minutes per group (Supplementary Figure 1). Upon joining a group, they started interacting with one partner in that group until the participant decided to leave the interaction and 'travel' to interact with a different partner in the group. The interactions took the form of a repeated economic game, approximating the dictator game, with the participant in the role of receiver. Thus, every 3.5 seconds the participant saw how their partner chose to share a new pot of credits, with varying stake sizes (Fig.1). All accumulated credits were added to the participant's bank, which the participants were told would be converted to a bonus payment at the end of the task.

Participants were told that decisions were collected from participants of a previous study. The decisions were in fact pre-coded by the researchers. The proportion shared by each partner decreased over time, representing a deterioration in the 'quality' of the interaction, as indexed by its fairness - 
defined as the proportion of the total pot shared, with $50 \%$ being completely fair and $0 \%$ completely unfair. The participant could leave the interaction at any time by pressing the spacebar. Following a leave decision there was an eight second delay prior to joining the next partner in the group, during which time participants received no credits, but were informed how many credits they had banked with the group so far. Participants interacted with each group for a total of five minutes. At the end of those five minutes they joined a completely new group of potential partners. When joining each new group, participants were explicitly told what type of group they were joining, and this information was represented throughout their time with each group by the colour of the screen's border (details for each study outlined below).

Crucially, while the 'fairness' of each partner's decisions decreased over the course of the interaction, the absolute value of the credits received by the participant on each trial was uniformly distributed around a constant. As such, regardless of the decay in fairness of a partner, or the fairness of others in the group, the average reward obtained was stable across sharing decisions in the experiment. Therefore, the economically rational behaviour in this task is to never leave a partner, unless fairness decays to $0 \%$, as during the eight seconds it takes to connect to another partner there is no financial reward received. Participants were informed that each partner had a finite number of decisions to make, and that if the participant did not leave an interaction they would automatically travel to the next partner when all decisions had been seen, incurring the eight second travel cost. The number of decisions coded of each partner was 30 , but participants were not instructed of this. Below we outline task details and variations across the four studies.

\section{Studies 1 and 2}

The aim of study 1 was to examine whether the value of a partner and the opportunity cost of the environment influenced how long people spend in a social interaction, and study 2 aimed to replicate this effect. Studies 1 and 2 used the same version of the task. The fairness of partners was manipulated by changing the rate of decay of the fairness of their sharing decisions, and the opportunity cost was manipulated by changing the proportion of fairer players (players with lower fairness decay rates) in two different social environments. A richer social environment, where the majority of partners had the low decay rate, and a poorer social environment where the majority had the higher decay rate. Thus, studies one and two had $2 \times 2$ designs, in which we could examine the effect of partner fairness (fair or unfair) and social environment quality (rich or poor) on how long people spent connected to the players before deciding to leave.

The proportion shared by each partner followed an exponential decay, as shown in equation 1. 
491

492

493

494

495

496

497

498

499

500

501

502

503

504

505

506

507

508

509

510

511

512

513

514

515

516

517

518

519

520

521

where $p_{t}$ is the proportion shared on trial $t$ and $k$ is the exponential decay rate. $s$, the starting value, was set to 0.5 (an equal split) for the purpose of creating the trajectory but the proportion shared of the first decision was replaced by selecting from a random uniform distribution with lower and upper bounds of 0.475 and 0.525 . Partners differed in the rate of exponential decay in their levels of fairness. There were two partner types, fair and unfair, with decay rates of -0.125 and -0.2 respectively (Fig.1). When the proportion dropped below 0.05 , this was replaced with a zero. This has the advantage of ensuring that participants could be excluded for failing to attend, as participants should leave if they are consistently receiving none of the pot. The absolute value of each sharing decision (the credits given to the participant) was randomly sampled from a uniform distribution with lower and upper bounds of 50 and 200, with the constraint that the total sum over six decisions must equal 500 . This constraint was included to ensure that, as close as possible, there would be no economically rational reason to prefer one partner over another, regardless of when one chose to leave. Stake sizes were determined by the quotient of the absolute value and proportion shared. Where these stake values equalled zero due to the fairness trajectory dropping to zero, the stake was drawn from a random uniform distribution with lower and upper bounds of 200 and 2000.

The environment manipulation in this version of the task was the average generosity of the group. When joining a new group, participants were explicitly told "Most partners shared a [high/low] proportion". Participants were instructed that the partners in each environment were randomly drawn from a large set of possible partners. The average generosity of the subset was then computed and compared to the overall average proportion shared. High generosity therefore meant that the average generosity of the group was greater than the average generosity overall. High generosity groups had a fair-to-unfair partner ratio of 3:1, although partners were not instructed of this ratio. Thus, in every set of four consecutive partners, participants would see three fair partners and one unfair partner (randomly ordered). The reverse was true for low generosity groups. This ensured that, as closely as possible, regardless of how many partners a participant interacted with during the five minutes, the experienced group generosity matched the 3:1 ratio of the group.

Participants joined eight groups in total, with four being high, and four being low generosity, making the overall time spent on the task 40 minutes. Partner decisions were created according to the parameters described above depending on their status as fair or unfair, and were pseudo-randomly assigned to groups. This ensured that all participants experienced the same eight groups, but the order in which they saw the groups was fully randomised across participants. 
Sharing decisions were shown in the middle of the screen (e.g. "98 out of 250") for 3.5 seconds before immediately showing the next sharing decision. When participants chose to leave one partner they were shown a countdown of eight seconds prior to connecting with the next partner.

\section{Study 3}

The environment manipulation in this study was the effort required to travel between partners. Previous research has shown that repeated finger movements are considered costly, and people will avoid them, unless associated with beneficial outcomes ${ }^{73,74}$. Here, effort was operationalised through repeated key presses during the 8 second delay. There were two environments, low and high effort. Effort levels were participant-specific. Prior to receiving any instructions on the task, participants were asked to press the right arrow key as many times as possible in 8 seconds. They were asked to do this three times, and the average taken as their maximal effort. The required number of button presses was $20 \%$ and $90 \%$ of their maximum for the low and high effort environments, respectively. This ensured that participants with greater or lesser fidelity at button presses did not consider the button presses too easy or beyond their capacity to complete. Unless otherwise stated, all other elements of this experiment were consistent with that of studies one and two.

In this study the ratio of fair to unfair partners was 1:1, making all social environments equally generous overall. The decay rates were the same as those used for Study 1 and 2. However, in this version of the task the proportion shared never dropped to zero. Instead, all proportions below 0.05 were replaced with 0.05 . This was to remove the potential confound that any partner effect could be due to economic rationality.

Participants joined eight groups in total, with four being high, and four being low effort, making the overall time spent on the task 40 minutes. Partner decisions were created according to the parameters described above depending on their status as fair or unfair, and were pseudo-randomly assigned to groups. This ensured that all participants experienced the same eight groups, but the order in which they saw the groups was fully randomised across participants.

\section{Study 4}

The social environment manipulation in this study was average generosity, as per Studies 1 and 2. However, to examine whether results would be consistent when partners showed a much greater range in behaviour, instead of having just two decay rates, the version used in this study had 17 different decay rates and noise was injected around each sharing decision. Decay rates for fair partners came from the set of $\{-0.075,-0.080,-0.085,-0.090,-0.095,-0.100,-0.105,-0.110,-0.115\}$, while those for unfair partners came from the set of $\{-0.175,-0.170,-0.165,-0.160,-0.155,-0.150$, - 

four consecutive partners containing three decay rates randomly drawn from the set of fair decay rates and one from the set of unfair decay rates. The opposite was true for low generosity environments. In this version of the task, the proportion of the first sharing decision was drawn from a random uniform distribution with lower and upper bounds of 0.47 and 0.53 . Each subsequent decision was dictated by the exponential decay associated with the specific partner, but with added noise as shown in Equation 2 below. This noise was added to each decisiondecision of eacheach partner meaning that the trajectory of each partner's sharing decisions was unique and less predictable. All proportions below 0.05 were replaced by 0.05 . The absolute value of credits shared on each decision was sampled from a random normal distribution with a mean of 400 and a standard deviation of 30 .

$$
p_{t}=s * e^{(k * t)}+\delta
$$

$$
\delta \sim N(0,0.015)
$$

In this version of the task, three of the first five partners of each environment were randomly selected to include an attention check. This ensured that even those who experience fewer partners in an environment still encountered these attention checks. For these three partners, participants were asked to press a key between partner decisions once in the first four trials with that partner. They were given 2.5 seconds to do so. Failure to successfully complete an attention check held no immediate repercussions for the participant, but missed attention checks informed exclusion criteria when analysing the data.

Visual representation of the sharing decisions in this version differed to previous versions (See Figure $1 \mathrm{~A}$ for details). In order to reduce any potential cognitive load of calculating the proportion shared, the sharing decisions were represented with a horizonal rectangle rather than numerically. The overall size of the rectangle represented overall stake available to the partner for that sharing decision, and a shaded area represented the proportion of the stake shared. This rectangle appeared on the screen for 1 second, followed by a blank screen for a period of time jittered around mean 2.5 seconds, thus making the average time of each trial 3.5 seconds as per the other versions of the task. decisions were created according to the parameters described above depending on their status as fair 
experienced the same ten groups, but the order in which they saw these groups was randomised across participants.

In Studies 1-3, participants attended in-person. They were first shown visual and text instructions for the task before discussing those with the researcher to ensure comprehension. They then completed a practice run which involved spending two minutes in each type of social environment, ensuring that any potential effects of the first environment experienced were minimised ${ }^{36}$. Following successful completion of the task training, participants completed the real version of the task.

Study 4 recruited participants online through Prolific. In addition to the task described above, participants in this study also completed the Depression Anxiety and Stress Scales (DASS-21) ${ }^{3}$ and the De Long Gierveld Loneliness Scale (DGLS) ${ }^{59}$. The order in which they completed the task and questionnaires was counterbalanced across participants. Following the task instructions, participants were asked a series of comprehension questions. If any questions were answered incorrectly, twice, they were excluded from the analysis $(n=3)$. Following completion of the comprehension questions, participants completed a two-minute practice run in each type of social environment before proceeding to the task. Upon completion of the task they answered some short debrief questions.

The task was coded in PsychoPy version 2020.1.37, and implemented through a Windows 10 PC (Studies 1 -3) or hosted on Pavlovia (pavlovia.org) (Study 4).

Statistical analysis

\section{Statistical models}

All statistical analyses were carried out using $R$ version $4 \cdot 0 \cdot 2^{76}$. All models were analysed as linear mixed-effects models using the packages Ime4, ImerTest, and car, with post-hoc analyses carried out with emmeans ${ }^{77-80}$. Data were visualised using the package ggplot2 and model visualisations used the package interactions ${ }^{81,82}$.

To test our hypotheses, we defined the same model for Studies $1-3$. The time at which the participant chose to leave an interaction ('leaving time' (LT)) was defined as a continuous outcome variable. If a participant chose not to leave a partner, the LT for that trial was defined as the onset of the final sharing decision (but see exclusion criteria below). Partner and environment types were defined as fixed-effects categorical predictors, with two levels each. We modelled both the main effects and the interaction term. We included a participant-level random intercept, as well as random slopes for each main effect (see Equation 3). The model did not converge when including the interaction term in the 
random effect structure due to a lack of variance across participants. Contrasts were effect-coded such that the intercept beta represented the grand mean, and the main effect betas represent the effect averaged over the levels of the other main effect. We tested the fixed-effects for statistical significance using a Type II Wald chi-square test. In the text we report the results of the chi-square test as well as the beta values and their $t$-test against zero.

$$
\text { LT } \sim \text { partner } * \text { environment }+(1+\text { partner }+ \text { environment } \mid I D)
$$

Due to Study 4 including multiple decay rates, the partner predictor was a continuous predictor (zscored decay rate), rather than categorical. The model was the same as that described above in all other respects. To test for relationships between task behaviour, depression and loneliness, we performed two additional mixed-effects models. These models were the same as for the main analysis, with the addition of depression or loneliness scores as z-scored continuous predictors. We tested for all main effects, 2-way interactions, and three-way interactions. It was not possible to include both loneliness and depression within the same model, as scores were too highly correlated (Spearman's rho $=0.65$ ) to examine unique variance associated with each.

In this task, not leaving a partner is an economically rational response. However, not actively choosing to leave a partner could also be a sign of inattention. Therefore, in Studies $1-3$, we implemented an exclusion criterion that participants must have made an equal number of active decisions as there were environments. While this may remove participants from the analysis who legitimately chose to never leave a partner, it is a minimal criterion in order to ensure some engagement in the task (Study 1 excluded $n=1$; Study 3 excluded $n=1$ ). Study 4 included attention checks. Participants were excluded if they failed more than $25 \%$ of attention checks $(n=21)$. For all studies, we excluded trials where the LT was more than 2.5 standard deviations of the mean, on a within-participant, withincondition basis. Again, this was to protect against lapses in attention.

\section{Sample size justification}

We ran the statistical model on 5000 simulated datasets based on the fixed effects and variance of the random effects from the analysis of Study 1. Power for each fixed effect was calculated as the proportion of simulations where $p<0.05$. This analysis suggested data from 25 participants would give us over $90 \%$ power to detect main effects of partner and environment type, and over $80 \%$ power to detect an interaction, with alpha at 0.05 . Therefore, we set this as our target sample size for Studies 2 and 3. We were uncertain as to a likely effect size for the exploratory analyses examining the role of depressive symptoms in Study 4, so aimed to recruit 100 participants for this study. 
We modelled decisions to leave as an evidence accumulation process (EA) process using a drift diffusion model $(D D M)^{83}$. We adapted this EA process based on a recent study ${ }^{40}$ that posited that animals make decisions to leave patches (locations) when reward foraging through an EA process that drifts towards leaving throughout a series of events of receiving rewards. This model therefore assumes that an animal will leave a patch when a noisy estimate of the state of the current value of rewards being obtained reaches a threshold. Here, instead of reward, we hypothesised that evidence accumulation would depend on the fairness of each decision from the partner that would guide decisions of when to leave a social interaction. To test this we deployed a model where evidence was based on the proportion shared in each decision from the partner, and compared this two other models, a standard model in which the evidence was accumulated regardless of the decisions by the partner, and a reward based model in which the magnitude of a reward influenced a decrease in evidence to leave.

The basic process is described by the equation:

$$
\mathrm{dEA}=(\mathrm{k}-\mathrm{X}) \mathrm{dt}+N(0, \sigma)
$$

$$
E A(t) \geq 0 \forall t
$$

664

where $\mathrm{X}$ is a variable that changes over the course of the accumulation. We ran three instantiations of the model which differed by the identity of the input variable $X . X$ could be either fairness, reward, or neither of them. The term ( $\mathrm{k}-\mathrm{X})$ drives the accumulation, $k$ is a parameter that modulates the input, and $N(0, \sigma)$ is a Gaussian noise term with standard deviation $\sigma$. We used $\mathrm{d} t=0.001 \mathrm{~s}$ and assumed that the model makes a decision to leave when the evidence is greater than the threshold. i.e. $|E A|>\theta$ (the decision threshold - once reached the participant leaves). We also assumed subjects might have some prior bias toward leaving, represented by a starting bias $\varepsilon . \theta, k, \sigma$, and $\varepsilon$ are free parameters of the model that were fit separately to each participants

Each version of the model was fitted to the individual participant's leaving time (LT) data using maximum likelihood estimation. Specifically, LT distributions were computed for each participant and condition. This LT distribution was compared to the LT generated by the model through repeated simulations. For a given set of parameter estimates, we estimated the log likelihood (LL) of the data using the following formula:

$$
L L=\sum_{i_{\text {cond }}=1}^{2} \log \left(K S\left(L T_{\text {data }}^{i_{\text {cond }}}, L T_{\text {model }}^{i_{\text {cond }}}\right)\right)
$$


where $\mathrm{KS}(p, q)$ estimates the probability that two distributions are equal according to the KolmogorovSmirnov test (computed using MATLAB function ktest2 which in turn estimates the predicted cumulative probability through the proportions of the predicted LTs which are less than or equal to any observed LT), i_cond is an index representing the condition (the generosity of the social environment). For each participant separately, we identified the set of model parameters that maximized the $L L$, by searching over a grid of values: $\theta=\{$ int 1:30 $\}, k=\{0.1: 1.5$, in steps of 0.1$\}, \sigma=$ $\{0.1: 1$, in steps of 0.1$\}$ and $\varepsilon=\{0: 0.8$, in steps of 0.2$\}$. These ranges were defined after an initial exploratory analysis over a wider range of parameter values to ensure selecting the ones that produced LT distributions spanning those seen in behaviour. For each set of parameters, we generated LT distributions by running 1,000 simulations of the model (that is, by producing this number of decision trajectories using equation (4) for each environment condition). To further assess the quality of the fits resulting from the best set of participant-specific parameters (those that maximized the LL function in equation (5)), we computed correlation coefficients between the average LT from the data and the model for all participants and conditions.

We ran three versions of the model where $\mathrm{X}$ would be equal, respectively to

$$
\begin{aligned}
& X(t)=F(t) \\
& X(t)=a * R(t) \\
& X(t)=0
\end{aligned}
$$

Where $a$ was a parameter which was scaling $R$ to match the range of values taken by $F$. As $F \in(0,1)$ it followed that $a=\max (R)=0.01$. The model with $X$ described by equation (8) was fitting LT distributions without any input from the task variables, offering a benchmark to compare the other models.

We compared the different models computing summed BIC. As a validation of the model comparison, we also looked at the proportion of subjects for which any given model was outperforming the others. Reassuringly, the winning model was also the one the most frequently explained the data better across the population (Supplementary Figure 2).

\section{Acknowledgements}

A.S.G was funded by an ESRC Postdoctoral Research Fellowship (ES/S011676/1). M.A.J.A was funded by a Biosciences and Biotechnology Research Council (BBSRC) Future Leader Fellowship (BB/M013596/1) and a BBSRC David Phillips Fellowship (BB/R010668/1). K.C.O was supported by a scholarship from the Marshall Aid Commemoration Commission. The work reported here was also 
709 supported by the John Fell Fund and the Christ Church Research Centre. We would like to thank Dr.

710 Patricia Lockwood for her comments on a draft of this manuscript, and members of the Motivation

711 and Social Neuroscience Lab for their ongoing feedback during the course of the project.

\section{Author contributions}

713 Conceptualisation by A.S.G and M.A.J.A, who also drafted the manuscript. A.S.G, M.A.J.A, A.P and 714 K.C.O edited the manuscript. Experimental design by A.S.G, M.A.J.A and K.C.O. Data analysis by A.S.G 715 and A.P. Data collection by A.S.G, K.C.O, and R.L. Supervision by M.A.J.A and A.S.G. Funding acquired 716 by M.A.J.A, A.S.G, and K.C.O.

\section{Data and code availability}

718 Analysis scripts and study data will be made available on the Open Science Framework upon 719 publication.

720 


\section{References}

1. Kupferberg, A., Bicks, L. \& Hasler, G. Social functioning in major depressive disorder. Neurosci. Biobehav. Rev. (2016) doi:10.1016/j.neubiorev.2016.07.002.

2. Robson, S. E., Repetto, L., Gountouna, V.-E. \& Nicodemus, K. K. A review of neuroeconomic gameplay in psychiatric disorders. Mol. Psychiatry 1 (2019) doi:10.1038/s41380-019-0405-5.

3. Henry, J. D. \& Crawford, J. R. The short-form version of the Depression Anxiety Stress Scales (DASS21): Construct validity and normative data in a large non-clinical sample. Br. J. Clin. Psychol. 44, $227-239$ (2005).

4. de Jong Gierveld, J., Van Tilburg, T. \& Dykstra, P. A. Loneliness and social isolation. Camb. Handb. Pers. Relatsh. 485-500 (2006).

5. Bangee, M., Harris, R. A., Bridges, N., Rotenberg, K. J. \& Qualter, P. Loneliness and attention to social threat in young adults: Findings from an eye tracker study. Personal. Individ. Differ. 63, 1623 (2014).

6. Kemperman, A., van den Berg, P., Weijs-Perrée, M. \& Uijtdewillegen, K. Loneliness of older adults: social network and the living environment. Int. J. Environ. Res. Public. Health 16, 406 (2019).

7. Umberson, D. \& Karas Montez, J. Social Relationships and Health: A Flashpoint for Health Policy. Journal of Health and Social Behavior vol. 51 S54-S66 (2010).

8. Crockett, M. J., Clark, L., Hauser, M. D. \& Robbins, T. W. Serotonin selectively influences moral judgment and behavior through effects on harm aversion. Proc. Natl. Acad. Sci. 107, 17433-17438 (2010).

9. Gabay, A. S., Kempton, M. J., Gilleen, J. \& Mehta, M. A. MDMA Increases Cooperation and Recruitment of Social Brain Areas When Playing Trustworthy Players in an Iterated Prisoner's Dilemma. J. Neurosci. 39, 307-320 (2019).

10. Gabay, A. S., Radua, J., Kempton, M. J. \& Mehta, M. A. The Ultimatum Game and the brain: A meta-analysis of neuroimaging studies. Neurosci. Biobehav. Rev. 47, 549-558 (2014). 
11. Güth, W., Schmittberger, R. \& Schwarze, B. An experimental analysis of ultimatum bargaining. J. Econ. Behav. Organ. 3, 367-388 (1982).

12. King-Casas, B. et al. The Rupture and Repair of Cooperation in Borderline Personality Disorder. Science 321, 806-810 (2008).

13. Oosterbeek, H., Sloof, R. \& Kuilen, G. van de. Cultural Differences in Ultimatum Game Experiments: Evidence from a Meta-Analysis. Exp. Econ. 7, 171-188 (2003).

14. Raihani Nichola J. \& Barclay Pat. Exploring the trade-off between quality and fairness in human partner choice. R. Soc. Open Sci. 3, 160510 (2016).

15. Rilling, J. K. \& Sanfey, A. G. The Neuroscience of Social Decision-Making. Annu. Rev. Psychol. 62, 23-48 (2011).

16. Camerer, C. F. Progress in behavioral game theory. J. Econ. Perspect. 11, 167-188 (1997).

17. Mastroianni, A. M., Gilbert, D. T., Cooney, G. \& Wilson, T. D. Do conversations end when people want them to? Proc. Natl. Acad. Sci. 118, e2011809118 (2021).

18. Sommerfeld, R. D., Krambeck, H.-J., Semmann, D. \& Milinski, M. Gossip as an alternative for direct observation in games of indirect reciprocity. Proc. Natl. Acad. Sci. 104, 17435-17440 (2007).

19. Dunbar, R. I., Marriott, A. \& Duncan, N. D. Human conversational behavior. Hum. Nat. 8, 231-246 (1997).

20. Schroeder, J. Ending Conversation is a Fraught Endeavor. Trends Cogn. Sci. 25, 552-553 (2021).

21. Charnov, E. L. Optimal foraging, the marginal value theorem. Theor. Popul. Biol. 9, 129-136 (1976).

22. Mobbs, D., Trimmer, P. C., Blumstein, D. T. \& Dayan, P. Foraging for foundations in decision neuroscience: insights from ethology. Nat. Rev. Neurosci. 19, 419 (2018).

23. Becker, S. W., Ronen, J. \& Sorter, G. H. Opportunity Costs-An Experimental Approach. J. Account. Res. 12, 317-329 (1974).

24. Niv, Y., Daw, N. D., Joel, D. \& Dayan, P. Tonic dopamine: opportunity costs and the control of response vigor. Psychopharmacology (Berl.) 191, 507-520 (2007). 
25. Kurzban, R., Duckworth, A., Kable, J. W. \& Myers, J. An opportunity cost model of subjective effort and task performance. Behav. Brain Sci. 36, 661-679 (2013).

26. Constantino, S. \& Daw, N. D. Learning the opportunity cost of time in a patch-foraging task. Cogn. Affect. Behav. Neurosci. 15, 837-853 (2015).

27. Le Heron, C. et al. Dopamine Modulates Dynamic Decision-Making during Foraging. J. Neurosci. 40, 5273-5282 (2020).

28. Gabay, A. S. \& Apps, M. A. J. Foraging optimally in social neuroscience: computations and methodological considerations. Soc. Cogn. Affect. Neurosci. (2020) doi:10.1093/scan/nsaa037.

29. Yoo, S. B. M., Hayden, B. Y. \& Pearson, J. M. Continuous decisions. Philos. Trans. R. Soc. B 376, 20190664 (2021).

30. Stephens, D. W. \& Krebs, J. R. Foraging Theory. (Princeton University Press, 1986).

31. Cassini, M. H., Lichtenstein, G., Ongay, J. P. \& Kacelnik, A. Foraging behaviour in guinea pigs: further tests of the marginal value theorem. Behav. Processes 29, 99-112 (1993).

32. Krebs, J. R., Ryan, J. C. \& Charnov, E. L. Hunting by expectation or optimal foraging? A study of patch use by chickadees. Anim. Behav. 22, 953-IN3 (1974).

33. Lewis, A. R. Selection of Nuts by Gray Squirrels and Optimal Foraging Theory. Am. Midl. Nat. 107, 250-257 (1982).

34. Hassell, M. P. \& Southwood, T. R. E. Foraging Strategies of Insects. Annu. Rev. Ecol. Syst. 9, 75-98 (1978).

35. Hayden, B. Y., Pearson, J. M. \& Platt, M. L. Neuronal basis of sequential foraging decisions in a patchy environment. Nat. Neurosci. 14, 933-939 (2011).

36. Garrett, N. \& Daw, N. D. Biased belief updating and suboptimal choice in foraging decisions. Nat. Commun. 11, 3417 (2020).

37. Yoon, T., Geary, R. B., Ahmed, A. A. \& Shadmehr, R. Control of movement vigor and decision making during foraging. Proc. Natl. Acad. Sci. 115, E10476-E10485 (2018). 
38. Goldfarb, A. \& Prince, J. Internet adoption and usage patterns are different: Implications for the digital divide. Inf. Econ. Policy 20, 2-15 (2008).

39. Lindström, B. et al. A computational reward learning account of social media engagement. Nat. Commun. 12, 1311 (2021).

40. Davidson, J. D. \& Hady, A. E. Foraging as an evidence accumulation process. PLOS Comput. Biol. 15, e1007060 (2019).

41. Kilpatrick, Z. P., Davidson, J. D. \& Hady, A. E. Normative theory of patch foraging decisions. ArXiv200410671 Math Q-Bio (2020).

42. Santini, Z. I., Koyanagi, A., Tyrovolas, S., Mason, C. \& Haro, J. M. The association between social relationships and depression: A systematic review. J. Affect. Disord. 175, 53-65 (2015).

43. Hirschfeld, R. M. et al. Social functioning in depression: a review. J. Clin. Psychiatry 61, 268-275 (2000).

44. Bangee, M., Harris, R. A., Bridges, N., Rotenberg, K. J. \& Qualter, P. Loneliness and attention to social threat in young adults: Findings from an eye tracker study. Personal. Individ. Differ. 63, 1623 (2014).

45. Schinka, K. C., van Dulmen, M. H., Mata, A. D., Bossarte, R. \& Swahn, M. Psychosocial predictors and outcomes of loneliness trajectories from childhood to early adolescence. J. Adolesc. 36, 12511260 (2013).

46. Cacioppo, S. et al. Loneliness and implicit attention to social threat: A high-performance electrical neuroimaging study. Cogn. Neurosci. 7, 138-159 (2016).

47. Stek, M. L. et al. Is Depression in Old Age Fatal Only When People Feel Lonely? Am. J. Psychiatry $162,178-180$ (2005).

48. Matthews, T. et al. Loneliness and neighborhood characteristics: A multi-informant, nationally representative study of young adults. Psychol. Sci. 30, 765-775 (2019).

49. Engel, C. Dictator games: a meta study. Exp. Econ. 14, 583-610 (2011). 
50. Henrich, J. et al. 'Economic man' in cross-cultural perspective: behavioral experiments in 15 smallscale societies. Behav. Brain Sci. 28, 795-815; discussion 815-855 (2005).

51. Kahneman, D., Knetsch, J. L. \& Thaler, R. H. Fairness and the Assumptions of Economics. J. Bus. 59, S285-S300 (1986).

52. The Handbook of Experimental Economics. (1997).

53. Chaudhuri, A. Sustaining cooperation in laboratory public goods experiments: a selective survey of the literature. Exp. Econ. 14, 47-83 (2011).

54. Charnov, E. L. OPTIMAL FORAGING, MARGINAL VALUE THEOREM. Theor. Popul. Biol. 9, 129-136 (1976).

55. Bernstein, C., Kacelnik, A. \& Krebs, J. R. INDIVIDUAL DECISIONS AND THE DISTRIBUTION OF PREDATORS IN A PATCHY ENVIRONMENT .2. THE INFLUENCE OF TRAVEL COSTS AND STRUCTURE OF THE ENVIRONMENT. J. Anim. Ecol. 60, 205-225 (1991).

56. Chong, T. T.-J. et al. Neurocomputational mechanisms underlying subjective valuation of effort costs. PLoS Biol. 15, e1002598 (2017).

57. Contreras-Huerta, L. S., Pisauro, A. \& Apps, M. A. Effort shapes social cognition and behaviour: A neuro-cognitive framework. Neurosci. Biobehav. Rev. (2020).

58. Lockwood, P. L. et al. Prosocial apathy for helping others when effort is required. Nat. Hum. Behav. 1, s41562-017-0131 (2017).

59. De Jong Gierveld, J. \& Van Tilburg, T. The De Jong Gierveld short scales for emotional and social loneliness: tested on data from 7 countries in the UN generations and gender surveys. Eur. J. Ageing 7, 121-130 (2010).

60. Turrin, C., Fagan, N. A., Monte, O. D. \& Chang, S. W. C. Social resource foraging is guided by the principles of the Marginal Value Theorem. Sci. Rep. 7, 11274 (2017).

61. Hunter, L. E. \& Daw, N. D. Context-sensitive valuation and learning. Curr. Opin. Behav. Sci. 41, 122$127(2021)$. 
62. Hayden, B. \& Niv, Y. The case against economic values in the orbitofrontal cortex (or anywhere else in the brain). (2020) doi:10.31234/osf.io/7hgup.

63. Krajbich, I., Hare, T., Bartling, B., Morishima, Y. \& Fehr, E. A common mechanism underlying food choice and social decisions. PLoS Comput. Biol. 11, e1004371 (2015).

64. Gold, J. I. \& Shadlen, M. N. The neural basis of decision making. Annu Rev Neurosci 30, 535-574 (2007).

65. Mante, V., Sussillo, D., Shenoy, K. V. \& Newsome, W. T. Context-dependent computation by recurrent dynamics in prefrontal cortex. Nature 503, 78-84 (2013).

66. Hutcherson, C. A., Bushong, B. \& Rangel, A. A Neurocomputational Model of Altruistic Choice and Its Implications. Neuron 87, 451-462 (2015).

67. Apps, M. A., McKay, R., Azevedo, R. T., Whitehouse, H. \& Tsakiris, M. Not on my team: Medial prefrontal cortex responses to ingroup fusion and unfair monetary divisions. Brain Behav. 8, e01030 (2018)

68. Wright, N. D., Symmonds, M., Fleming, S. M. \& Dolan, R. J. Neural segregation of objective and contextual aspects of fairness. J. Neurosci. Off. J. Soc. Neurosci. 31, 5244-5252 (2011).

69. Andersen, S., Ertaç, S., Gneezy, U., Hoffman, M. \& List, J. A. Stakes Matter in Ultimatum Games. Am. Econ. Rev. 101, 3427-3439 (2011).

70. Harman, G. What is moral relativism? in Values and morals 143-161 (Springer, 1978).

71. Harman, G. \& Thomson, J. J. Moral relativism and moral objectivity. (1996).

72. Mastroianni, A. M., Gilbert, D. T., Cooney, G. \& Wilson, T. D. Do conversations end when people want them to? Proc. Natl. Acad. Sci. 118, (2021).

73. Bächinger, M. et al. Human motor fatigability as evoked by repetitive movements results from a gradual breakdown of surround inhibition. Elife 8, e46750 (2019).

74. Contreras-Huerta, L. S., Lockwood, P. L., Bird, G., Apps, M. A. \& Crockett, M. J. Prosocial behavior is associated with transdiagnostic markers of affective sensitivity in multiple domains. Emotion (2020). 
873

874

875

876

877

878

879

880

881

882

883

884

885

886

887

888

889

75. Peirce, J. et al. PsychoPy2: Experiments in behavior made easy. Behav. Res. Methods 51, 195-203 (2019).

76. $\mathrm{R}$ Development Core Team. R: A language and environment for statistical computing. ( $R$ Foundation for Statistical Computing, 2020).

77. Bates, D., Mächler, M., Bolker, B. \& Walker, S. Fitting Linear Mixed-Effects Models Using Ime4. J. Stat. Softw. 67, 1-48 (2015).

78. Kuznetsova, A., Brockhoff, P. B. \& Christensen, R. H. B. ImerTest Package: Tests in Linear Mixed Effects models. J. Stat. Softw. 82, 1-26 (2017).

79. Lenth, R. V. emmeans: Estimated Marginal Means. (2020).

80. Fox, J. \& Weisberg, S. An $\{R\}$ Companion to Applied Regression. (Sage).

81. Long, J. A. interactions: Comprehensive, User-Friendly Toolkit for Probing Interactions. (2019).

82. Wickham, H. ggplot2: Elegant Graphics for Data Analysis. (Springer-Verlag, 2016). doi:10.1007/978-0-387-98141-3.

83. Ratcliff, R., Smith, P. L., Brown, S. D. \& McKoon, G. Diffusion Decision Model: Current Issues and History. Trends Cogn. Sci. 20, 260-281 (2016). 


\section{Supplementary methods}

Participant (green figure) connects with a partner
in a social environment. While connected they
repeatedly receive credits based on that partners
sharing decisions. At any time the participant can
choose to leave that partner. When they do so
they spend 8 seconds travelling to the next
partner in the environment, during which time
they receive no credits.
Partners differed by their fairness, here indicated
by the colour of the potential partners. By
changing the proportion of purple to blue
partners, we could manipulate the environment's
average generosity. For example, on the left
there are more purple (fair) than blue (unfair)
partners. After 5 minutes, participants joined a
totally different group of potential partners

Studies 1 \& 2 group manipulation: average generosity

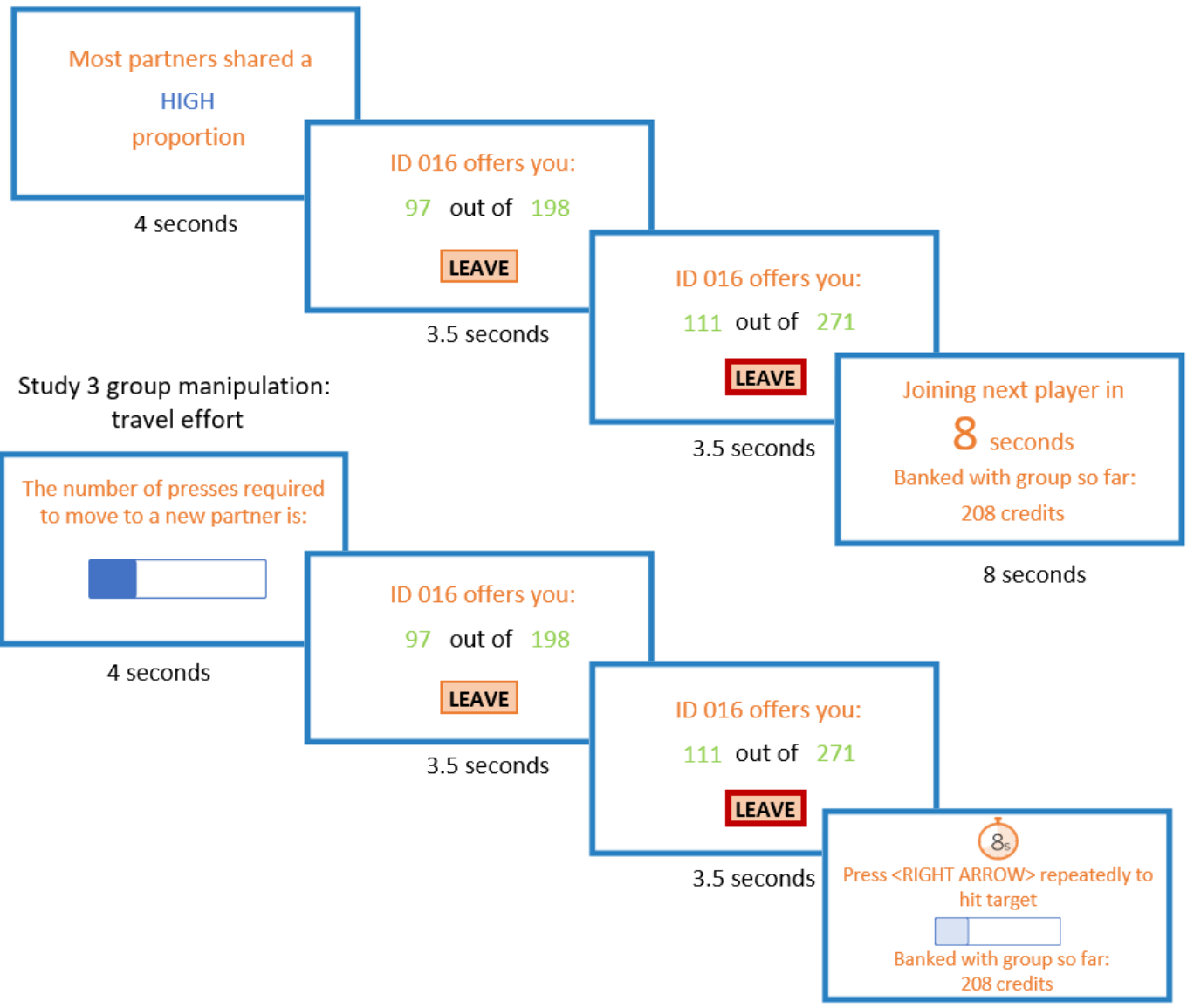

8 seconds 
899 Supplementary Table 1: Demographics by study, after exclusions.

\begin{tabular}{lllll}
\hline & Study 1 & Study 2 & Study 3 & Study 4 \\
\hline$N$ & 19 & 25 & 24 & 81 \\
$\% F$ & 76.4 & 65.4 & 73.1 & 49.4 \\
Age & 27.6 (SD 5.3) & 23.7 (SD 4.5) & 24.2 (SD 5.0) & 30.7 (SD 8.5) \\
Environment manipulation & Generosity & Generosity & Effort & Generosity \\
\hline
\end{tabular}

900

901 
904 Control analysis (Study 1 \& 2) - excluding zero fairness trials

905 The fairness trajectories used in Study 1 and Study 2 (see methods for details) allowed for fairness to 906 drop to zero. Continuing to interact with a partner while they share no money is a legitimate behaviour 907 in the task, but it could also be a sign of inattention. We repeated the analyses from both study 1 and 908 study 2, excluding decisions made after the fairness had dropped to zero. The main effects of partner 909 and environment type remained statistically significant for both studies, while the interaction 910 between predictors was non-significant for both studies (Supplementary Table 1).

Supplementary Table 2: Results from linear mixed models for Study 1 \& Study 2 when excluding decisions when fairness was equal to zero

\begin{tabular}{|l|l|l|l|l|l|l|l|}
\hline Study & Predictor & $b$ & SE & $t$ & $p$-value & $X^{2}$ & $p$-value \\
\hline 1 & Partner & -8.21 & 0.77 & -10.619 & $<0.001$ & 113.36 & $<0.001$ \\
\hline & Environment & 2.53 & 0.78 & 3.237 & 0.001 & 11.01 & 0.001 \\
\hline 2 & $\begin{array}{l}\text { Partner*environment } \\
\text { interaction }\end{array}$ & -2.00 & 1.54 & -1.297 & 0.195 & 1.68 & 0.195 \\
\hline & Partner & -10.15 & 1.21 & -8.400 & $<0.001$ & 70.98 & $<0.001$ \\
\hline & Environment & 2.52 & 1.13 & 2.221 & 0.044 & 4.87 & 0.027 \\
\hline & $\begin{array}{l}\text { Partner*environment } \\
\text { interaction }\end{array}$ & 1.03 & 1.56 & 0.658 & 0.51 & 0.43 & 0.510 \\
\hline
\end{tabular}

913 
916 We examined whether the fairness at the time of choosing to leave a partner differed by partner type 917 or social environment for each study. Across all studies there was a main effect of both partner type 918 and social environment, and no interaction. In all cases, the fairness at leaving was lower for less fair 919 partners than fairer partners, and in low quality environments compared to high.

920 Supplementary Table 3: Results from linear mixed models for all studies, with fairness as the outcome variable

\begin{tabular}{|l|l|l|l|l|l|l|l|}
\hline Study & Predictor & $b$ & SE & $t$ & $p$-value & $X^{2}$ & $p$-value \\
\hline 1 & Partner & -2.63 & 0.63 & -4.146 & 0.001 & 16.82 & $<0.001$ \\
\hline & Environment & -2.08 & 0.90 & -2.316 & 0.032 & 5.48 & 0.019 \\
\hline & $\begin{array}{l}\text { Partner*environment } \\
\text { interaction }\end{array}$ & 1.46 & 0.94 & 1.56 & 0.120 & 2.42 & 0.120 \\
\hline 2 & Partner & -3.15 & 0.75 & -4.170 & $<0.001$ & 17.40 & $<0.001$ \\
\hline & Environment & -2.68 & 0.92 & 2.914 & 0.008 & 8.51 & 0.004 \\
\hline 3 & $\begin{array}{l}\text { Partner*environment } \\
\text { interaction }\end{array}$ & -0.58 & 0.99 & -0.588 & 0.557 & 0.35 & 0.557 \\
\hline & Partner & -1.99 & 0.37 & -5.311 & $<0.001$ & 27.95 & $<0.001$ \\
\hline & Environment & -1.23 & 0.38 & -3.268 & 0.001 & 10.48 & 0.001 \\
\hline & $\begin{array}{l}\text { Partner*environment } \\
\text { interaction }\end{array}$ & -0.40 & 0.75 & -0.537 & 0.591 & 0.29 & 0.591 \\
\hline & $\begin{array}{l}\text { Decay rate (partner } \\
\text { type) }\end{array}$ & 0.02 & 0.001 & 10.877 & $<0.001$ & 120.33 & $<0.001$ \\
\hline & Environment & -0.01 & 0.004 & -2.142 & 0.035 & 4.54 & 0.033 \\
\hline & $\begin{array}{l}\text { Partner*environment } \\
\text { interaction }\end{array}$ & -0.004 & 0.002 & -1.724 & 0.085 & 2.97 & 0.085 \\
\hline
\end{tabular}

921 
924 We examined whether the amount earned per partner differed by partner type or social environment 925 for each study. Across all studies there was a main effect of both partner type and social environment. 926 In all cases, participants earned less with unfair partners than fairer partners, and earned more in low 927 quality environments than high quality environments. Since participants accumulate reward while 928 interacting with a partner, and earn no reward while travelling between partners, this pattern of results is to be expected. Leaving times were earlier in a high opportunity cost environment, meaning more time was spent travelling, and therefore less reward earned. People spent more time interacting with fair partners than unfair partners, and therefore earned more money with those partners. There was a partner by environment interaction in Study 1, but this did not replicate across studies.

933 Supplementary Table 4: Results from linear mixed models for all studies, with earnings as the outcome variable

\begin{tabular}{|l|l|l|l|l|l|l|l|}
\hline Study & Predictor & $b$ & SE & $t$ & $p$-value & $X^{2}$ & $p$-value \\
\hline 1 & Partner & -343.28 & 40.68 & -8.44 & 0.001 & 71.84 & $<0.001$ \\
\hline & Environment & 72.42 & 32.11 & 2.255 & 0.037 & 5.23 & 0.022 \\
\hline & $\begin{array}{l}\text { Partner*environment } \\
\text { interaction }\end{array}$ & -71.00 & 29.94 & -2.371 & 0.018 & 5.62 & 0.018 \\
\hline & Partner & -331.19 & 36.38 & -9.103 & $<0.001$ & 82.86 & $<0.001$ \\
\hline & Environment & 78.98 & 29.58 & 2.670 & 0.14 & 7.11 & 0.008 \\
\hline 3 & $\begin{array}{l}\text { Partner*environment } \\
\text { interaction }\end{array}$ & -13.99 & 30.87 & -0.453 & 0.651 & 0.21 & 0.650 \\
\hline & Partner & -214.50 & 33.59 & -6.385 & $<0.001$ & 41.51 & $<0.001$ \\
\hline 4 & Environment & 145.77 & 33.63 & 4.334 & $<0.011$ & 18.50 & $<0.001$ \\
\hline & $\begin{array}{l}\text { Partner*environment } \\
\text { interaction }\end{array}$ & 40.72 & 67.05 & 0.607 & 0.544 & 0.37 & 0.544 \\
& $\begin{array}{l}\text { Decay rate (partner } \\
\text { type) }\end{array}$ & 758.15 & 60.79 & 12.472 & $<0.001$ & 156.44 & $<0.001$ \\
\hline & Environment & 324.72 & 106.27 & 3.056 & 0.003 & 9.38 & 0.002 \\
\hline & $\begin{array}{l}\text { Partner*environment } \\
\text { interaction }\end{array}$ & -51.24 & 55.59 & -0.922 & 0.357 & 0.85 & 0.357 \\
\hline
\end{tabular}

934

Supplementary analysis - do depression/loneliness scores predict earnings?

To test whether the altered behaviour seen in participants with higher self-reported depression and loneliness was more economically rational, we carried out two regression analyses predicting overall earnings by depression or loneliness. Supplementary Table 5 shows that neither of these predictors were significant.

Supplementary Table 5: Results from regression analyses testing the relationship between overall task earnings and depression/loneliness

\begin{tabular}{|l|l|l|l|l|}
\hline Predictor & $b$ & SE & $t$ & $p$-value \\
\hline Depression & 1069 & 3307 & 77.229 & 0.749 \\
\hline Loneliness & 225.5 & 1805.9 & 0.125 & 0.901 \\
\hline
\end{tabular}


946

947

948

949

950

951

952

953

954

955

Supplementary Table 6: mixed model results for simulated data from non-winning DDMs
\begin{tabular}{|l|l|l|l|l|l|l|l|}
\hline Model & Predictor & $b$ & SE & $t$ & $p$-value & $X^{2}$ & $p$-value \\
\hline Reward & Partner & 0.002 & .014 & 0.012 & 0.990 & 0.46 & 0.495 \\
\hline & Environment & -3.07 & 0.14 & -21.705 & $<0.001$ & 975.86 & $<0.001$ \\
\hline & $\begin{array}{l}\text { Partner*environment } \\
\text { interaction }\end{array}$ & -0.1 & 0.20 & -0.694 & 0.488 & 0.48 & 0.488 \\
\hline $\begin{array}{l}\text { Standard } \\
\text { DDM }\end{array}$ & Partner & 0.05 & 0.14 & 0.383 & 0.702 & 1.09 & 0.297 \\
\hline & Environment & -0.07 & 0.14 & -0.575 & 0.565 & 0.10 & 0.751 \\
\hline & $\begin{array}{l}\text { Partner*environment } \\
\text { interaction }\end{array}$ & 0.10 & .019 & 0.496 & 0.620 & 0.25 & 0.620 \\
\hline
\end{tabular}

Supplementary Figure 2: Percentage of participants with each model as their winning model. The fairness adapted DDM was the best fitting model in $65 \%$ of participants

\section{Mixed effect models on the non-winning modelled-simulated data}

To further demonstrate that the reward model and standard DDM did not capture the shape of the experimental data we ran the statistical model on the simulated dataset from these models. Supplementary Table 4 shows how neither model captures both the partner-type and social environment effect present in the real data.

Supplementary Table 6: mixed model results for simulated data from non-winning DDMs

\section{Fairness model}

Reward model

Standard DDM 

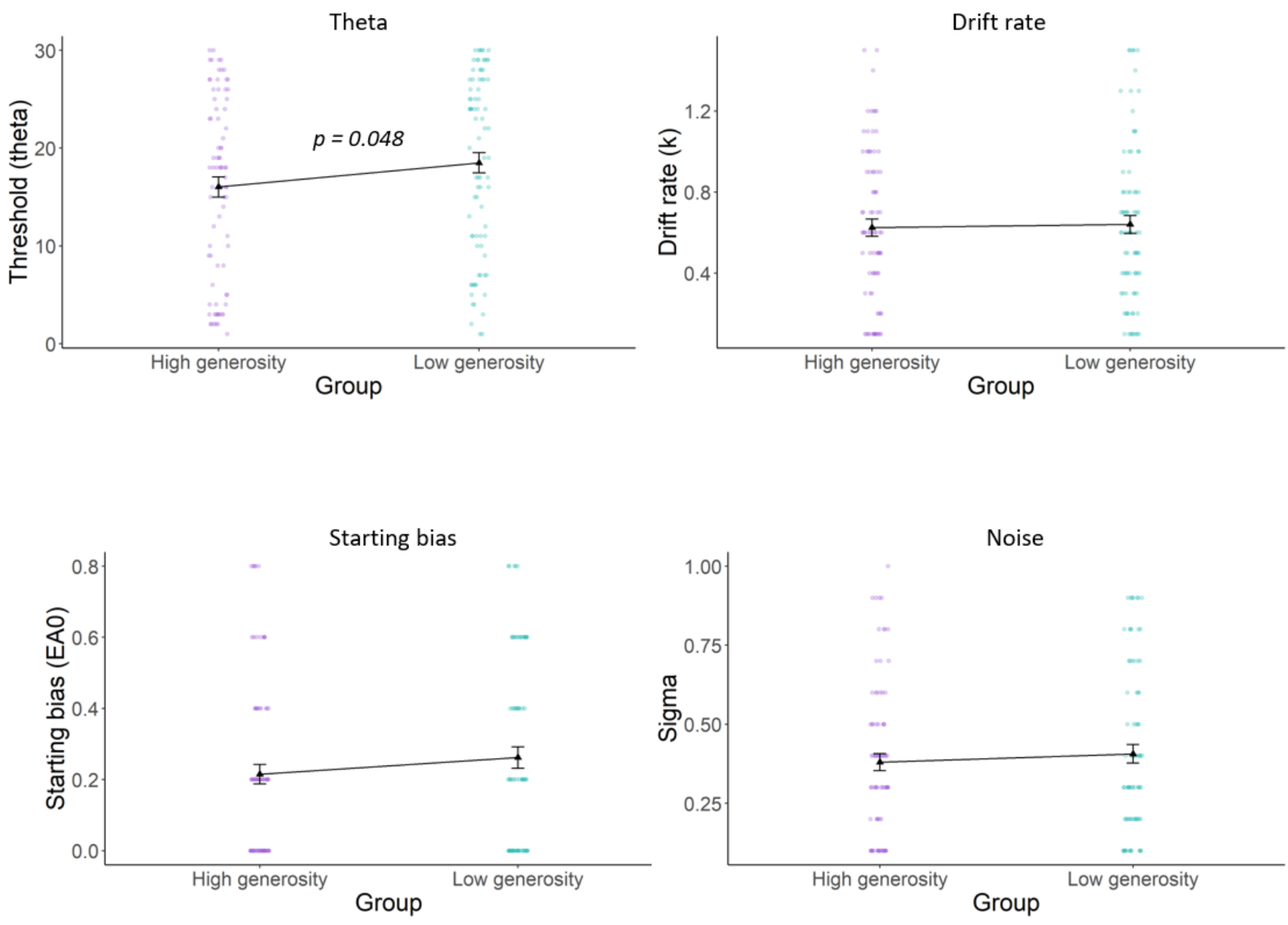

959 Supplementary Figure 3: fitted parameter values for the winning Fairness DDM. The threshold parameter, theta differed 960 across environments. All other parameters did not differ significantly between social environments 